\title{
Ambiguity And Social Interaction*
}

\author{
Jürgen Eichberger \\ Alfred Weber Institut, \\ Universität Heidelberg.
David Kelsey
Department of Economics, University of Exeter. \\ Burkhard C. Schipper ${ }^{\dagger}$ \\ Department of Economics, \\ University of California, Davis.
}

30th March 2007

\begin{abstract}
We present a non-technical account of ambiguity in strategic games and show how it may be applied to economics and social sciences. Optimistic and pessimistic responses to ambiguity are formally modelled. We show that pessimism has the effect of increasing (decreasing) equilibrium prices under Cournot (Bertrand) competition. In addition the effects of ambiguity on peace-making are examined. It is shown that ambiguity may select equilibria in coordination games with multiple equilibria. Some comparative statics results are derived for the impact of ambiguity in games with strategic complements.
\end{abstract}

Keywords: Ambiguity, Optimism, Pessimism, Strategic Games, Oligopoly, Strategic Delegation, Peace-making, Strategic Complements, Choquet Expected Utility.

JEL Classification: C72, D43, D62, D81.

*Financial support by ESRC grant ES-000-22-0650 (David) and the DFG, SFB/TR 15 (Burkhard) is gratefully acknowledged. We thank Sujoy Mukerji, Jeffrey Kline, Simon Grant, Frank Milne, Wei Pang, Marzia RaybaudiMassilia, Kirk McGrimmon, Peter Sinclair and Willy Spanjers as well as seminar participants at the Australian National University, the Universities of Alabama, Birmingham, Berlin (Humboldt), Edinburgh, Exeter, the Royal Economic Society and the EEA for helpful comments.

${ }^{\dagger}$ Corresponding author: Department of Economics, University of California, Davis, One Shields Avenue, Davis, CA 95616, USA, Tel: +1-530-752 6142, Fax: +1-530-752 9382, Email: bcschipper@ucdavis.edu 


\section{Introduction}

The growing literature on ambiguity in strategic games lacks two important features: First, an elementary framework of ambiguity in games that can be easily understood and applied also by non-specialists, and second, the treatment of an optimistic attitude towards ambiguity in addition to ambiguity aversion or pessimism. In this article we take up both issues.

Ambiguity or Knightian uncertainty refers to a situation where probabilities are unknown or imperfectly know as opposed to a situation under risk where probabilities are known. This distinction has been made by Knight (1921). In fact for many economic or political situations under uncertainty, it is not obvious at all why decision makers should know probabilities. For instance, threats from terrorism and rogue states, and the likely impact of new technologies, etc. Nevertheless, for several decades, subjective expected utility (henceforth SEU) by Savage (1954) appeared to have rendered the distinction between risk and Knightian uncertainty obsolete. In this theory, individuals faced with uncertainty behave as if they hold beliefs that can be represented by a subjective probability distribution. Hence, from an analytical point of view, there was little distinction between risk and ambiguity.

However early evidence by Ellsberg (1961) suggests that beliefs cannot be represented by conventional probabilities. Systematic laboratory experiments have confirmed Ellsberg's conjecture, see Camerer and Weber (1992). Despite the experimental evidence, SEU proved to be a successful modelling tool. Important insights were obtained from the distinction between risk preferences and beliefs, which can be made in this approach. The economics of insurance and information could be developed in this context. It is desirable to develop a theory of ambiguity, which is equally suitable for application. The inconsistencies between Savages's theory and empirically observed behaviour have stimulated efforts for alternative theories. One of the most promising of these is Choquet expected utility (henceforth CEU), which involves representing individuals' beliefs by non-additive probabilities (or capacities, i.e., probabilities that do not necessarily add up to one), see Schmeidler (1989). In this theory, individuals maximise the expected value of a utility function with respect to a non-additive belief, and the expectation

is expressed as a Choquet integral, Choquet (1953-4). CEU is a generalization of subjective expected utility. It has the advantage that it maintains the separation of beliefs and outcome evaluation, which makes the theory easier to apply in economics and social sciences. 
A key element of Knight's work was that people differ in their attitudes to ambiguity. The majority of people tend to avoid ambiguous situations. However a minority of individuals actually appear to seek ambiguity. Experimental evidence shows a similar pattern. A majority of experimental subjects behave more cautiously when probabilities are undefined, while a significant minority displays the opposite attitude, (see for instance Camerer and Weber (1992)). Moreover the same individual may be pessimistic (or ambiguity averse) in one situation and optimistic (or ambiguity seeking) in another. The evidence shows that those ambiguity attitudes are distinct from risk attitudes, see Cohen, Jaffray, and Said (1985). Individuals may be risk-averse and ambiguity-loving and vice-versa.

Most of the work on Choquet expected utility theory has been focused on arbitrary convex capacities, i.e., capacities that allow for a pessimistic attitude to ambiguity only. Consequently, the generality of arbitrary convex capacities made expositions relatively inaccessible to the nonspecialist and the focus on the pessimistic attitude limited the scope of potential applications. Recently, Chateauneuf, Eichberger, and Grant (2007) axiomatised Choquet expected utility theory, where preferences are represented as a weighted average of the expected utility, the maximum utility and the minimum utility. While a special case, this representation has the advantage of being simple to apply and easy to interpret. Moreover, it allows for a meaningful notion of both optimism as well as pessimism as weight put on the best and worst outcomes respectively. We take this model further and apply it to strategic games.

In strategic games we model interaction among players who face ambiguity about the opponent's strategy. A player may react optimistically (resp. pessimistically) towards this ambiguity by over-weighting strategies of the opponent which are good (resp. bad) to the player. We introduce the concept of Equilibrium under Ambiguity, prove existence and relate it to Nash equilibrium without ambiguity. An Equilibrium under Ambiguity is an equilibrium when beliefs are represented by capacities.

We demonstrate the simple applicability of our framework and present some comparative static results on the impact of ambiguity. Specifically we consider the impact of optimism and pessimism in some familiar models from industrial organisation. In a Cournot oligopoly with linear demand and constant marginal cost, the worst (resp. best) outcome would be perceived as a rival producing a large (resp. low) quantity. Under these assumptions, Cournot oligopoly is a game of strategic substitutes and negative externalities. Optimism (resp. pessimism) has 
the effect of increasing (resp. reducing) the perceived marginal benefit of producing more and so increases (resp. reduces) the equilibrium output. This decreases (resp. raises) profits but increases (resp. reduces) consumer surplus. In contrast, in Bertrand competition, a good (resp. bad) outcome would be perceived as rival firms charging a high (resp. low) price. Typically there is strategic complementarity in Bertrand models. In this case, optimism (resp. pessimism) will increase (resp. reduce) the incentive for any given firm to increase its price and hence will also increase (resp. reduce) the equilibrium price.

Traditionally, it has been suspected that oligopolies are prone to informal collusive arrangements. Scherer (1970) provides many examples from anti-trust cases. The presumption of regulators that oligopolists collude, suggests that output is, at least sometimes, below the Cournot level without clear evidence of collusion. Ambiguity-aversion may offer an alternative and as yet unexplored explanation for why competition may be less fierce in Cournot-style oligopoly than predicted by Nash equilibrium.

In Cournot and Bertrand models, there is scope for strategic delegation. Interestingly, in both Bertrand and Cournot oligopoly we show that it is desirable for the owner of a firm to delegate decision-making to a manager who is more optimistic than (s)he is. This result is rather striking since usually comparative results are reversed in one setting versus the other. Already Knight (1921) argued in his theory of profit and entrepreneurial activity, that entrepreneurs tend to be individuals who are less ambiguity-averse. Indeed, there is evidence from interviews with new entrepreneurs that their self-assessed chances of success are uncorrelated with objective predictors like education, prior experience, and initial capital, and are in average widely off the mark (Cooper, Dunkelberg, and Woo (1988)). In a different study, new life insurance agents who were optimistic sold more policies during their first year and were less likely to quit (Seligman and Schulman (1986)).

Ambiguity is not confined to economic situations. For instance, environmental risks are often ambiguous due to limited knowledge of the relevant science and because outcomes will only be seen many decades from now. The effects of global warming and the environmental impact of genetically modified crops are two examples. Other fields characterized by ambiguity are politics, diplomacy and international security as many recent events in world politics dramatically demonstrate. To illustrate the potential of our framework we consider a stylized model of peace-making in section 5. Because of the singularity of each conflict, it is hard for involved 
parties for come up with exact probability judgements. Thus ambiguity plays a large role in conflict resolution such as in the Middle East peace-making (see for example Klieman (1999))1 or the conflict in Northern Ireland. This is confirmed by statements of key players involved in peace-making, such as Garret Fitzgerald, former Taoiseach of the Republic of Ireland, who said that "(f)rom the start to finish a striking feature of the whole prolonged Peace Process has been the prevalence of ambiguity in every aspect what has been done. Ambiguity about the relationship between the IRA and Sinn Fein, ambiguity about the commitment to decommissioning; ambiguity about the meaning of the ceasefire; ambiguity between successive Irish governments and Sinn Fein. The last truth is that without all this literally demoralising ambiguity we would not have come to the point where 35 years of continuous violence in the North and 80 years of sporadic violence in our own State that has cost the lives of politicians and many members of the security forces is about to end." (Irish Times, 12th April 2003). The role of ambiguity in peace-making is largely circumstantial. Ambiguity leaves room for the optimistic hope that peace indeed will be accomplished but pessimistic participants may also respond to ambiguity with distrust in the opponent. We find that in our model these ambiguity-attitudes can determine the success or failure of a peace process.

Beside above mentioned applications, we provide several new general results. For 2-player games with real-valued strategy space, we show that if ambiguity is sufficiently large, then equilibrium under ambiguity is unique. If in addition the game has strategic complements we provide new results on the comparative statics of equilibria with respect to optimism and pessimism. For such games with multiple equilibria we show results on equilibrium selection due to sufficiently large degrees of optimism or pessimism.

The article is organized as follows: Section 2 describes how to model ambiguity in strategic games. Then in section 3 we discuss a solution concept for games, which allows for the possibility that the behaviour of other players may be perceived to be ambiguous. We show existence and the relationship with Nash equilibrium. In section 4 we demonstrate with oligopoly models the simple applicability of our framework to economics. Non-economic applications are demonstrated by the model of peace-making in section 5. Some general results concerning the

\footnotetext{
${ }^{1}$ For example Kissinger remarked about the US brokered August 1970 cease-fire along the Suez Canal "Born in fateful ambiguity", see Klieman (1999). More recently, the Los Angeles Times (May 28, 2003) carried an article "Road Map Torn by Ambiguity" by Max Abrahms from The Washington Institute for Near East Policy.
} 
comparative statics of ambiguity are presented in section 6 and section 7 contains our conclusions. The appendix contains proofs of those results not proved in the text.

\section{Modelling Ambiguity in Strategic Games}

In this section we explain how ambiguity can be modelled in strategic games by non-additive beliefs. We present the concepts of a neo-additive capacity (a class of non-additive beliefs) and of the Choquet integral.

\subsection{Games}

Unless otherwise mentioned, we consider for simplicity a strategic game $G=\left\langle\left(S_{i}, u_{i}\right)_{i=1,2}\right\rangle$ with two players $i=1,2$, where each player's strategy set $S_{i} \subseteq \mathbb{R}$ is a closed and bounded real-valued interval. Economic examples often deal with quantities and prices. Hence, for most purposes, it is sufficient to assume that each agent chooses real-valued variables. The pay-off function of player $i$ denoted by $u_{i}\left(s_{i}, s_{-i}\right)$ is assumed to be quasi-concave in his/her strategy and twice continuously differentiable in strategies of both players. The following notational conventions will be maintained throughout this paper. The set of strategy combinations will be denoted by $S=S_{1} \times S_{2}$. A typical strategy combination $s \in S$ can be decomposed into the strategy $s_{i}$ of player $i$ and the strategy of the opposing player $s_{-i}, s=\left(s_{i}, s_{-i}\right)$. The set of strategy combinations of player $i$ 's opponent is denoted by $S_{-i}$.

\section{$2.2 \quad$ Non-Additive Beliefs and Expectations}

Consider an economic agent whose profit may depend in part on the actions of rivals. Here, ambiguity concerns the possible play of one's opponent. We shall represent individuals' beliefs by capacities. A capacity plays a similar role to a subjective probability in SEU. In this paper we shall confine attention to neo-additive capacities? defined below.

Definition 2.1 (Neo-additive Capacity) Let $\gamma, \lambda$ be real numbers such that $0 \leqslant \gamma \leqslant 1$, $0 \leqslant \lambda \leqslant 1-\gamma$, and let $\pi$ be a probability distribution on $S_{-i}$, define a neo-additive capacity $\nu$ by $\nu(A)=\lambda+(1-\lambda-\gamma) \pi(A), \emptyset \varsubsetneqq A \varsubsetneqq S_{-i} ; \nu(\emptyset)=0, \nu\left(S_{-i}\right)=1$.

\footnotetext{
${ }^{2} \mathrm{Neo}$ is an abbreviation for Non-extremal outcome additive. CEU with neo-additive capacities is axiomatised in Chateauneuf, Eichberger, and Grant (2007).
} 
Let $u$ be a utility function which represents the decision-makers' pay-offs as a function of the acts of his/her opponent. The expectation of $u$ with respect to the neo-additive capacity $\nu$, is given by the Choquet integral (see Chateauneuf, Eichberger, and Grant (2007)) $\mathrm{H}^{3}$

Definition 2.2 (Choquet Integral) The Choquet expected value of the utility $u_{i}$ with respect to the neo-additive capacity $\nu=\lambda+(1-\lambda-\gamma) \pi$ from playing $s_{i} \in S_{i}$ is given by:

$$
\int u_{i}\left(s_{i}, s_{-i}\right) d \nu=\lambda M_{i}\left(s_{i}\right)+\gamma m_{i}\left(s_{i}\right)+(1-\gamma-\lambda) \mathbf{E}_{\pi} u_{i}\left(s_{i}, s_{-i}\right)
$$

where $\mathbf{E}_{\pi} u_{i}\left(s_{i}, \cdot\right)$ denotes the expected utility of $u_{i}$ with respect to the probability distribution $\pi$ on $S_{-i}, M_{i}\left(s_{i}\right)=\max _{s_{-i} \in S_{-i}} u_{i}\left(s_{i}, s_{-i}\right)$, and $m_{i}\left(s_{i}\right)=\min _{s_{-i} \in S_{-i}} u_{i}\left(s_{i}, s_{-i}\right)$.

Beliefs modelled by neo-additive capacities have several advantages. First, they are easy to interpret. Intuitively a neo-additive capacity describes a situation in which the individual believes that the likelihood of events is described by the additive probability measure $\pi$. However (s)he lacks confidence in this belief. In part (s)he reacts to this in an optimistic way by overweighting the best outcome $M$ as measured by $\lambda$ and in part the reaction is pessimistic by over-weighting the worst outcome $m$, measured by $\gamma$. The amount of perceived ambiguity may be measured by the sum $\gamma+\lambda$.

Second, CEU with neo-additive capacities is easy to relate to some familiar decision rules:

1. If $\lambda=0$ and $\gamma=1$, preferences have the maximin form and are extremely pessimistic;

2. if $\lambda=1$ and $\gamma=0$, preferences exhibit the maximal degree of optimism;

3. if $\lambda+\gamma=1$, these preferences coincide with the Hurwicz criterion, (see Hurwicz (1951));

4. if $\lambda=\gamma=0$, beliefs coincide with conventional expectations. In particular the capacity is additive, which implies that for all events $A, B \subseteq S_{-i}, A \cap B=\emptyset, \nu(A \cup B)=\nu(A)+\nu(B)$, i.e., the capacity is a conventional probability distribution.

${ }^{3}$ Gilboa (1987), Schmeidler (1989) and Sarin and Wakker (1992) provide axiomatisations for general CEU preferences. Ghirardato and Marinacci (2002), Wakker (2001) and Epstein (1999) characterise capacities representing ambiguity-averse or pessimistic attitudes of a decision maker. There is also a closely related literature which represents beliefs as sets of conventional probability distributions, see Bewley (2002), Gilboa and Schmeidler (1989), Kelsey (1994). 
Third, neo-additive capacities reduce the technical complexity of the CEU model. The Choquet integral is similar to a conventional expectation since it is a weighted average of utilities. In particular it is a convex combination of the minimum, the maximum and the average payoff. More important, neo-additive capacities are less mathematically complex than more general classes of capacities, i.e., capacities that satisfy just monotonicity, $A \subseteq B, A, B \subseteq S_{-i}$, $\Rightarrow$ $\nu(A) \leq \nu(B)$, and normalization, $\nu(\emptyset)=0$ and $\nu\left(S_{-i}\right)=1$.

One of the reasons why CEU with general capacities (see Schmeidler, 1989) is often hard to apply is because capacities may involve many parameters. While only $n-1$ parameters will describe a probability distribution on a set with $n$ elements, a general capacity on the same set involves $2^{n}$ parameters. In contrast, a neo-additive capacity can be described by just $n+1$ parameters.

Fourth, there is experimental evidence that preferences have the form of a weighted average of the minimum, the maximum and some mean pay-offs, see Lopes (1987) $4^{4}$

Finally, CEU with neo-additive capacities allows us to define a meaningful notion of optimism and pessimism (attitude towards ambiguity), as well as lack of confidence in the belief (ambiguity).

Definition 2.3 (Optimism/Pessimism) Let $\nu=\lambda+(1-\lambda-\gamma) \pi$ be a neo-additive capacity. We define the degree of optimism (resp. pessimism) of $\nu$ by $\lambda(\nu)=\lambda,($ resp. $\gamma(\nu)=\gamma)$.

One can interpret the additive part of a neo-additive capacity $\pi$ as the decision-maker's belief and $(1-\lambda-\gamma)$ as the degree of confidence in that belief. In the light of equation (1), we refer to the parameter $\lambda$ (resp. $\gamma$ ) as degree of optimism (resp. pessimism). If beliefs are represented by conventional probabilities, it is not possible to model decision-makers who lack confidence in their beliefs. The ability to make this distinction offers opportunities to analyse the impact of ambiguity and optimism/pessimism in economic models. With neo-additive capacities it is relatively easy to study the comparative statics with respect to ambiguity-attitudes.

The Choquet integral is similar to a conventional expectation since it is a weighted average of utilities and the weights sum to 1 . However the weights are not probabilities but decision weights. Neo-additive capacities allow us to model optimistic or pessimistic individuals according to the decision weights which they apply to outcomes. The best (resp. worst) outcome, $M$ (resp. $m$ )

\footnotetext{
${ }^{4}$ Such preferences have been axiomatised in the context of risk by Cohen (1992).
} 
gets weight $\lambda+(1-\gamma-\lambda) \pi(M)$ (resp. $\gamma+(1-\gamma-\lambda) \pi(m))$. For any other outcome $x$ the decision weight is $(1-\gamma-\lambda) \pi(x)$. The Choquet integral is simply the sum over all outcomes of the act weighted by these decision weights 5

If $\lambda=0$, then preferences may be represented in the form $a \succcurlyeq b \Leftrightarrow \min _{\pi \in C} \mathbf{E}_{\pi} u(a) \geqslant$ $\min _{\pi \in C} \mathbf{E}_{\pi} u(b)$, where $C$ is a set of conventional additive probabilities. We believe this formula is intuitive. When a decision-maker does not know the true probability (s)he considers a set of probabilities to be possible. He/she behaves cautiously and evaluates any course of action by the least favourable probability distribution. This small deviation from subjective probabilities allows us to capture the certainty effect ${ }^{6}$, which is consistently observed in experimental work (Gonzalez and Wu (1999) and Kilka and Weber (1998)).

Finally, we need to define the support of a capacity.

Definition 2.4 (Support) The support of the neo-additive capacity $\nu(A)=\lambda+(1-\lambda-\gamma) \pi(A)$, is defined by supp $\nu=\operatorname{supp} \pi$.

Note that contrary to probability distributions, for the support of a neo-additive capacity we have $\nu(\operatorname{supp} \nu)+\nu\left(S_{-i} \backslash \operatorname{supp} \nu\right)=1-\gamma+\lambda$, which is not in general equal to 1 . If beliefs are represented by a neo-additive capacity then the best and worst outcomes will influence choice in addition to members of the support.

\section{Equilibrium under Ambiguity}

In this section we present an equilibrium concept for strategic games with ambiguity. Consider player $i$ who faces ambiguity about his/her opponent's choice of strategy. If player $i$ 's beliefs are modelled by a neo-additive capacity $\nu_{i}$ on $S_{-i}$, i.e., with an additive probability distribution $\pi_{i}$ on $S_{-i}$, a degree of optimism $\lambda_{i}$ and a degree of pessimism $\gamma_{i}$, then the expected payoff function is the Choquet integral,

$$
V_{i}\left(s_{i} ; \pi_{i}, \lambda_{i}, \gamma_{i}\right)=\lambda_{i} M_{i}\left(s_{i}\right)+\gamma_{i} m_{i}\left(s_{i}\right)+\left(1-\lambda_{i}-\gamma_{i}\right) \int u_{i}\left(s_{i}, s_{-i}\right) d \pi_{i}\left(s_{-i}\right) .
$$

\footnotetext{
${ }^{5}$ Sarin and Wakker (1998) provide a detailed discussion of the relationship between decision weights and capacities.

${ }^{6}$ An individual displays the certainty effect if (s)he is willing to pay a large premium to avoid a small risk of receiving nothing.
} 
In games, one can determine $\pi_{i}$ endogenously as the prediction of the players from the knowledge of the game structure and the preferences of others. In contrast, we treat the degrees of optimism, $\lambda_{i}$ and pessimism, $\gamma_{i}$ as exogenous.

Define the best-response correspondence of player $i$ given that his/her beliefs are represented by a neo-additive capacity $\nu_{i}$ by $R_{i}\left(\nu_{i}\right)=R_{i}\left(\pi_{i}, \lambda_{i}, \gamma_{i}\right):=\arg \max _{s_{i} \in S_{i}} V_{i}\left(s_{i} ; \pi_{i}, \lambda_{i}, \gamma_{i}\right)$.

Definition 3.1 (Equilibrium under Ambiguity) A pair of neo-additive capacities $\left(\nu_{1}^{*}, \nu_{2}^{*}\right)$ is an Equilibrium Under Ambiguity (EUA) if for $i=1,2$,

$$
\operatorname{supp} \nu_{i}^{*} \subseteq R_{-i}\left(\nu_{-i}^{*}\right) .
$$

In equilibrium, each player assigns strictly positive likelihood to his/her opponent's best responses given the opponent's belief. However, each player lacks confidence in his/her likelihood assessment and responds in an optimistic way by over-weighting the best outcome, or in a pessimistic way by over-weighting the worst outcome. This notion of equilibrium is similar to that suggested by Dow and Werlang (1994) for 2-player games which was extended to n-player games by Eichberger and Kelsey (2000). Neither notion is more general than the other, since we allow for optimistic as well as pessimistic preferences while Dow and Werlang (1994) and Eichberger and Kelsey (2000) allow for arbitrary but convex capacities.

Our notion of Equilibrium under Ambiguity has a close relation to the standard Nash equilibrium. Indeed, for no ambiguity, $\lambda_{i}=\gamma_{i}=0$ for all $i=1,2$, this solution concept would coincide with Nash equilibrium of the game $G=\left\langle\left(S_{i}, u_{i}\right)_{i=1,2}\right\rangle$. Let $\pi^{*}=\left(\pi_{1}^{*}, \pi_{2}^{*}\right)$ be a (possibly mixed) Nash equilibrium, then $\pi_{i}^{*}$ is player's $i$ additive belief over the opponent $-i$ 's strategy with the property that any action in the support of $\pi_{i}^{*}$ is a best-response given $\pi_{-i}^{*}$. This is the interpretation of Nash equilibrium as equilibrium in beliefs. Above we define an Equilibrium under Ambiguity as an equilibrium in non-additive beliefs $\left(\nu_{1}^{*}, \nu_{2}^{*}\right)$. Equilibrium strategies are given by the supports of the capacities, which are required to be best-responses. If these are unique, we have a pure equilibrium. If there are several strategies, which a player considers as equal best, then any combination of these is possible in equilibrium. For example in Matching Pennies, any combination of "heads" and "tails" will represent equilibrium behaviour as long as both players do not believe that the opponent would favour a particular choice. If there is no ambiguity, then the equilibrium definition (3.1) specifies a pair of independent additive probability distributions, which is a mixed strategies Nash equilibrium. 
There is a second connection between the Equilibrium under Ambiguity and Nash equilibrium. Let $\lambda=\left(\lambda_{1}, \lambda_{2}\right)$ and $\gamma=\left(\gamma_{1}, \gamma_{2}\right)$, and consider the strategic game $\Gamma(\lambda, \gamma)=$ $\left\langle\left(S_{i}, V_{i}\left(\cdot ; \cdot, \lambda_{i}, \gamma_{i}\right)\right)_{i=1,2}\right\rangle$, i.e., the "perturbed" game derived from $G=\left\langle\left(S_{i}, u_{i}\right)_{i=1,2}\right\rangle$ by replacing $u_{i}$ with the Choquet expected payoff functions $V_{i}\left(\cdot ; \cdot, \lambda_{i}, \gamma_{i}\right)$ for $i=1,2$. We claim that any pure strategy Nash equilibrium $s^{*}=\left(s_{1}^{*}, s_{2}^{*}\right)$ of the perturbed game $\Gamma(\lambda, \gamma)$ is a pure strategy Equilibrium under Ambiguity $\nu^{*}=\left(\nu_{1}^{*}, \nu_{2}^{*}\right)$ of the game $G$ with degree of optimism $\lambda_{i}$ and degree of pessimism $\gamma_{i}$ for $i=1,2$. Indeed, for each player $i$ define a neo-additive capacity $\nu_{i}^{*}=\lambda_{i}+\left(1-\lambda_{i}-\gamma_{i}\right) \pi_{i}^{*}$ that assigns probability $\pi_{i}^{*}\left(s_{-i}^{*}\right)=1$ to $s_{-i}^{*}$. Since $\left(s_{1}^{*}, s_{2}^{*}\right)$ is a Nash equilibrium of $\Gamma(\lambda, \gamma)$, we have $\operatorname{supp}\left(\nu_{i}^{*}\right)=\left\{s_{-i}^{*}\right\} \subseteq R_{-i}\left(\nu_{-i}^{*}\right)$. Thus $\nu^{*}$ is an Equilibrium under Ambiguity. This proves the following proposition:

Proposition 3.1 For any pure strategy Nash equilibrium $s^{*}=\left(s_{1}^{*}, s_{2}^{*}\right)$ of $\Gamma(\lambda, \gamma)=$ $\left\langle\left(S_{i}, V_{i}\left(\cdot ; \cdot, \lambda_{i}, \gamma_{i}\right)\right)_{i=1,2}\right\rangle$, there is a pure strategy Equilibrium under Ambiguity (EUA) $\nu^{*}=$ $\left(\nu_{1}^{*}, \nu_{2}^{*}\right)$ of $G=\left\langle\left(S_{i}, u_{i}\right)_{i=1,2}\right\rangle$, in which player $i$ has degrees of optimism $\lambda_{i}$ and pessimism $\gamma_{i}$, and $\pi_{i}^{*}\left(s_{-i}^{*}\right)=1$.

This observation leads immediately to an existence result. In economic applications, players' strategy sets are mostly continuous variables, such as prices, quantities and investment expenditures. In such situations, pure Nash equilibria exist 7 To extend this idea to strategic games with ambiguity, we just need to ensure that the best (resp. worst) outcome is "well-behaved".

Definition 3.2 (Positive/Negative Externalities) The game $G$ has positive (resp. negative) externalities if $u_{i}\left(s_{i}, s_{-i}\right)$ is increasing (resp. decreasing) in $s_{-i}$, for $i=1,2$.

If there are positive (resp. negative) externalities a good outcome will be interpreted as the opponent playing a high (resp. low) strategy. Many games relevant to economics have such a property. Proposition 3.2 shows that for such games EUA can be applied whenever Nash equilibrium can.

\footnotetext{
${ }^{7}$ We shall not consider mixed strategies in the present paper. Even for additive beliefs, the interpretation of mixed Nash equilibria is debatable, see Osborne and Rubinstein (1994). If beliefs are strictly non-additive, then behaviour, whether in pure or mixed strategies, cannot coincide with the strategies played, since there are no non-additive randomising devices.
} 
Proposition 3.2 (Existence) If the game has positive or negative externalities, the strategy sets $S_{i} \subseteq \mathbb{R}$ are non-empty, compact and convex for all players $i=1,2$, and the payoff functions $u_{i}\left(s_{i}, s_{-i}\right)$ are continuous in $s$ and concave in each player's own strategy $s_{i}$ for any $s_{-i} \in S_{-i}$, then there exists an Equilibrium under Ambiguity (EUA) in pure strategies.

Proof. By Proposition 3.1, it suffices to show that a pure strategy Nash equilibrium of the game $\Gamma(\lambda, \gamma)$ exists. By standard arguments, i.e., Glicksberg (1952), $\Gamma(\lambda, \gamma)$ has a pure strategy Nash equilibrium if for $i=1,2, S_{i}$ is a non-empty compact convex subset of an Euclidian space and $V_{i}$ is continuous in $s$ and quasi-concave in $s_{i}$. We need to show that $V_{i}$ is quasi-concave in $s_{i}$, all other properties are straightforward.

Since $G$ has positive (resp. negative) externalities, $u_{i}$ is continuous in $s$ and $S_{-i} \subseteq \mathbb{R}$ is nonempty and compact, we have for any $s_{i}^{\prime \prime} \in S_{i}$ that $\arg \max _{s_{-i} \in S_{-i}} u_{i}\left(s_{i}^{\prime \prime}, s_{-i}\right) \ni \max S_{-i}$ (resp. $\left.\min S_{-i}\right)$ and $\arg \min _{s_{-i} \in S_{-i}} u_{i}\left(s_{i}^{\prime \prime}, s_{-i}\right) \ni \min S_{-i}\left(\operatorname{resp} . \max S_{-i}\right)$. Together with $u_{i}$ being concave in $s_{i}$ for each $s_{-i} \in S_{-i}$, it follows that $M_{i}$ and $m_{i}$ are concave in $s_{i}$. Finally, note that for any $\lambda_{i}, \gamma_{i}$ with $0 \leq \gamma_{i} \leq 1$ and $0 \leq \lambda_{i} \leq 1-\gamma_{i}, V_{i}$ is a positively weighted sum of functions concave in $s_{i}$, which implies that $V_{i}$ itself is concave in $s_{i}$. Thus $V_{i}$ is quasi-concave in $s_{i}$.

We illustrate Equilibrium under Ambiguity by the following example ${ }^{8}$

Example 1 There are two players, an incumbent monopolist, $I$, and an entrant, $E$. If the entrant chooses not to enter, ne, (s)he will receive payoff 0 and the incumbent will receive the monopoly profits $M$. If the entrant enters the market, $e$, the incumbent has the choice of accommodating entry, $a$ or fighting a price war, $f$. If the incumbent accommodates entry, both firms receive the duopoly profit $d$. Fighting entry causes both firms to sustain losses $-L$. The interaction between the incumbent and the entrant may be represented as the following normal form game:

Incumbent

\begin{tabular}{cc|c|c|} 
& & $a$ & $f$ \\
\cline { 2 - 4 } Entrant & $e$ & $d, d$ & $-L,-L$ \\
\cline { 2 - 4 } & ne & $0, M$ & $0, M$ \\
\hline
\end{tabular}

8 The example also shows that convex sets of strategies are not necessary in Proposition 3.2 
where $M>d>0$ and $L>0$.

There are two Nash equilibria (without ambiguity), $(a, e)$, and $(f, n e)$. In the first, the incumbent accommodates and the entrant enters, while in the second the incumbent fights and the entrant stays out. Based on standard equilibrium refinements such as subgame perfection in an appropriately defined extensive form game, it is common to regard the latter equilibrium as less plausible. Once the entrant is in the industry, the incumbent will make lower profits by fighting than by accommodating.

Now we shall consider how ambiguity affects this example. We shall assume that agents are purely pessimistic, i.e. $\gamma>0, \lambda=0$. When there is ambiguity, we find a new type of equilibrium. In this, the incumbent does not fight. However entry does not occur because the entrant is pessimistic and perceives considerable ambiguity about the incumbent's behaviour. Consider the following beliefs: $\nu^{E}(a)=\alpha, \nu^{E}(f)=0, \frac{L}{(d+L)}>\alpha>0, \nu^{I}(e)=0, \nu^{I}(n e)=\beta$, $1 \geqslant \beta>0$. These beliefs show a high degree of pessimism for the entrant. With these beliefs the (Choquet) expected payoff of the incumbent is given by:

$$
V^{I}(a)=M \beta+d(1-\beta), \quad V^{I}(f)=M \beta-L(1-\beta) .
$$

Hence, $a$ is a best response for the incumbent. If $\beta<1$, then $f$ is not a best response for the incumbent. Since this holds for all $\beta<1$, even small amounts of ambiguity-aversion are capable of eliminating non-credible threats.$^{9}$ The (Choquet) expected utility of the entrant is given by,

$$
V^{E}(e)=d \alpha-L(1-\alpha), \quad V^{E}(n e)=0 .
$$

Thus ne is a best response for the entrant if and only if,

$$
\alpha \leqslant \frac{L}{(d+L)}
$$

We interpret this as saying the entrant will not enter if (s)he is sufficiently ambiguity-averse. Equation (5) says that entry is more likely, the higher are the profits from successful entry $d$ and the lower are the losses from a price war, $L$. In Nash equilibrium, entry is independent of these factors provided $d$ and $L$ are both positive. In our opinion, it is not implausible that these factors would affect the outcome.

\footnotetext{
${ }^{9}$ This is true more generally see, Eichberger and Kelsey (2000), Proposition 5.1.
} 
The case of large ambiguity-aversion shows how deviations from Nash equilibrium can arise in EUA. The entrant considers it more likely that the incumbent will accommodate entry and this belief is sustained in equilibrium. It is possible that such a decision might be affected by ambiguity, since a firm will usually have much less information about an industry in which it does not already have a presence. In practice, entry is likely to entail considerable expenditure before any returns are received. By definition, the entrant is not already in the industry. Thus (s)he may face some considerable ambiguity about relevant variables, in particular the behaviour of the incumbent. It is not implausible that entrants might react by behaving cautiously and not entering even if they do not expect the incumbent to fight a price war.

In standard Nash theory, the assumption that the incumbent will be more likely to play $a$, implies that it is optimal to play $e$, which yields the higher payoff of $d$. This need not be the case in an EUA, if the entrant is sufficiently ambiguity-averse. Clearly, the possibility that the incumbent might fight entry, an event which is not in the support of the belief, influences the equilibrium outcome. Such behaviour is not implausible when players perceive ambiguity. If the entrant thinks that the incumbent will be cautious and accommodate, (s)he may still not be bold enough, to enter, since a misjudgment will earn him/her an outcome of $-L$.

Our intuition suggests that $(n e, a)$ is not an implausible way to behave. We suspect, however, that the degree of ambiguity-aversion depends upon observations. As evidence builds up that the opponent plays $a$ (or $f$ respectively), confidence may grow and choosing $e(n e)$ may become more likely. With a support notion, which insists that strategy combinations outside the support do not affect behaviour, $(n e, a)$ can never be an equilibrium. It seems to us an advantage of EUA, that it opens the possibility to model such testable hypothesis.

\section{Oligopoly Models}

In this section, we shall present some examples of how the techniques described in previous sections, can be used to examine the effect of ambiguity on economic behaviour. These examples will illustrate that the consequences of ambiguity can be examined without technical sophistication. 


\subsection{Cournot Oligopoly}

\subsubsection{Equilibrium under Ambiguity}

First we consider a symmetric Cournot duopoly, where firms produce homogenous products and choose quantities as their strategic variable. We will show that, in this case, optimism increases competition because it induces more aggressive behaviour. Pessimism will, in general, have the opposite effect.

There are two firms, $i=1,2$, which compete in quantities. Assume that firm $i$ faces the linear inverse demand curve $p_{i}\left(x_{i}, x_{-i}\right)=\max \left\{1-x_{i}-x_{-i}, 0\right\}$. We shall assume that each firm can produce at constant marginal cost equal to $c$. Firm $i$ chooses the quantity it wants to supply, $x_{i}$, from the interval $[0,1]$. If beliefs are represented by neo-additive capacities, a firm over-weights the best and worst outcomes. We assume that firm $i$ perceives the worst scenario to be a situation, where its rival dumps a large quantity on the market, driving the price down to zero. The firm's perceived best outcome is assumed to be where the rival produces zero output and the firm is a monopolist. Under these assumptions firm $i$ 's (Choquet) expected profit is:

$$
V_{i}\left(x_{i}, x_{-i}\right)=\lambda x_{i}\left(1-x_{i}\right)+\left(1-\gamma_{i}-\lambda_{i}\right) x_{i}\left[1-x_{-i}-x_{i}\right]-c x_{i} .
$$

A possible criticism of this model is that the choice of the best and the worst outcome is arbitrary. However our results remain true provided the best (resp. worst) outcome is below (resp. above) the Nash equilibrium output 10

The first order condition for maximising firm 1's profit is, $\frac{d V_{1}}{d x_{1}}=\lambda_{1}\left(1-2 x_{1}\right)+\left(1-\gamma_{1}-\lambda_{1}\right)$ $\left(1-2 x_{1}-x_{2}\right)=c$. Hence the reaction function of firm 1 is given by,

$$
R^{1}\left(x_{2}\right)=\frac{\left(1-\gamma_{1}\right)-\left(1-\gamma_{1}-\lambda_{1}\right) x_{2}-c}{2\left(1-\gamma_{1}\right)} .
$$

Proposition 4.1 In a symmetric equilibrium where $\lambda_{1}=\lambda_{2}=\lambda$ and $\gamma_{1}=\gamma_{2}=\gamma$, the equilibrium output and price are given by

$$
\bar{x}=\frac{1-\gamma-c}{3-3 \gamma-\lambda}, \quad \bar{p}=\frac{1-\gamma-\lambda+2 c}{3-3 \gamma-\lambda} .
$$

Proof. From equation $(7)$, the equilibrium output $\bar{x}$ is given by $\bar{x}=\frac{(1-\gamma)-(1-\gamma-\lambda) \bar{x}-c}{2(1-\gamma)}$, which implies $\bar{x}=\frac{1-\gamma-c}{3-3 \gamma-\lambda}$. The equilibrium price is given by $\bar{p}=\frac{1-\gamma-\lambda+2 c}{3-3 \gamma-\lambda}$.

\footnotetext{
${ }^{10}$ See Eichberger and Kelsey (2002) Proposition 3.1, for a related result which does not depend on assumptions about the perceived best and worst outcomes.
} 
The next result shows that if firms become more optimistic, (i.e., $\lambda$ increases) then equilibrium output will rise. An increase in optimism will increase the weight the firm puts on rivals producing a low output. This increases the marginal benefit of producing more and hence results in an increase in equilibrium output.

Proposition 4.2 The effects of changes in ambiguity attitude on equilibrium in Cournot oligopoly are as follows:

1. an increase in optimism increases output and decreases prices in equilibrium;

2. if $\lambda<3 c$, then an increase in pessimism reduces equilibrium output and increases prices.

Proof. By inspection, $\bar{x}$ is an increasing function of $\lambda$. Since $\frac{d p}{d \bar{x}}=-2$, the equilibrium price is a decreasing function of $\lambda$. The effect of an increase in pessimism on output is given by, $\frac{d \bar{x}}{d \gamma}=\frac{\lambda-3 c}{(3-3 \gamma-\lambda)^{2}}$, which is negative provided $\lambda<3 c$. As before, $\frac{d p}{d \gamma}=-2 \frac{d \bar{x}}{d \gamma}$.

Intuitively, more optimism causes a firm to place more weight on the possibility that its rival will produce a low output. This increases the marginal profitability of extra output. Thus the given firm will produce more. This reasoning is not restricted to the specific demand and cost functions but will apply whenever Cournot oligopoly is a game of strategic substitutes. In general one would not expect the effect of optimism to be large, hence it seems reasonable to assume $\lambda<3 c$, in which case an increase in pessimism would decrease equilibrium output. Likewise an increase in pessimism causes firms to place more weight on the possibility that a rival will produce a high output. This reduces the marginal benefit of producing more and hence tends to decrease equilibrium strategies 11

Corollary 4.1 Assume $\bar{x}>\frac{1}{4}(1-c)$, then:

1. an increase in optimism decreases equilibrium profits;

2. if $\lambda<3 c$, then an increase in pessimism increases equilibrium profits.

\footnotetext{
${ }^{11}$ However in this case there is an opposing effect. Assume cost is zero, by equation 6 the objective function of firm $i$ is cardinally equivalent to $\tilde{V}_{i}=\frac{\lambda}{1-\gamma} x_{i}\left(1-x_{i}\right)+\frac{(1-\gamma-\lambda)}{1-\gamma} x_{i}\left[1-x_{-i}-x_{i}\right]$. Increasing $\gamma$ increases the weight placed on the monopoly profits in this expression and hence increases the output. A similar effect applies when cost is low but not zero i.e. $3 c<\lambda$.
} 
Proof. Symmetric equilibrium profits are given by, $V=(p(x(\lambda))-c) x(\lambda)$, hence $\frac{\partial V}{\partial \lambda}=(p(\bar{x}(\lambda))-c) \frac{\partial \bar{x}}{\partial \lambda}+\frac{d p}{d \bar{x}} \frac{\partial \bar{x}}{\partial \lambda} \bar{x}(\lambda)=\frac{\partial \bar{x}}{\partial \lambda}\left[p(\bar{x}(\lambda))-c+\frac{d p}{d \bar{x}} \bar{x}(\lambda)\right]=\frac{\partial \bar{x}}{\partial \lambda}[1-c-4 \bar{x}(\lambda)]$. Thus provided $\bar{x}>\frac{1}{4}(1-c)$ an increase in $\lambda$ decreases profit. The proof of part 2 follows by similar reasoning.

The condition $\bar{x}>\frac{1}{4}(1-c)$ says that the effects of ambiguity are relatively small, in the sense that they do not induce firms to produce less than the collusive output. We would view this as the normal case.

To illustrate these results consider the case where there is no optimism $\lambda=0$. By equation (7) the symmetric equilibrium is characterised by

$$
\frac{1}{2}-\frac{3}{2} \bar{x}=\frac{1}{2(1-\gamma)} c
$$

Assume that there is an increase in pessimism, $\gamma$ rises. Then the rhs. of equation (8) increases. Since the lhs. of equation (8) is decreasing in $\bar{x}(\gamma), \bar{x}$ must be a decreasing function of $\gamma$. An increase in pessimism will decrease the quantities in a symmetric Cournot equilibrium, as depicted in Figure 1. As firms are symmetric, EUA are intersections of the best response function with the 45-degree line.

Figure 1: Cournot equilibrium and ambiguity

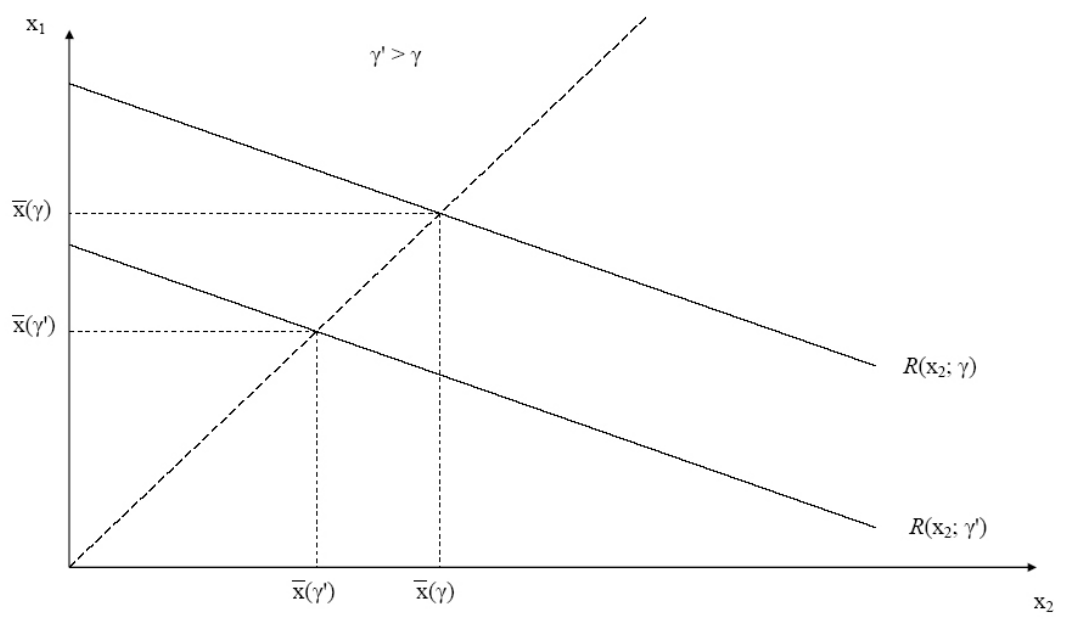

Pessimism reduces the amount brought to market. Intuitively, ambiguity makes a decisionmaker cautious about the behaviour of the opponent. By dumping output onto the market, the rival can drive down the price. If firms become more concerned about this possibility, they will 
reduce output in order to avoid the losses that would arise in such a case.

\subsubsection{Strategic Delegation}

In this section we show that it may be profitable to delegate decision-making to a manager who is more optimistic than the owner of the firm. This allows the owner to commit to producing a larger output, which is advantageous in a game of strategic substitutes. Hiring an optimistic manager has a similar effect to giving the manager an incentive to maximise a weighted sum of profits and revenue, as discussed in Vickers (1985).

Assume that firm 1 has a profit maximising owner who is ambiguity neutral, i.e., has additive beliefs. The owner hires a manager to operate the firm on his/her behalf. The owner pays him a wage, which is fraction $\alpha$ of firm 1's profit. The manager has CEU preferences and has beliefs represented by a neo-additive capacity. The owner chooses the manager to maximise his/her profit. Firm 2 is a conventional profit maximising firm. The following result finds the levels of optimism, $\lambda_{1}$ and pessimism, $\gamma_{1}$, which are optimal for the owner of firm 1.

Proposition 4.3 The profit maximising levels of $\lambda_{1}$ and $\gamma_{1}$ satisfy

$$
\lambda_{1}=1-3 \gamma_{1}+2 \gamma_{1} \frac{(1+c)}{(1-c)}
$$

Proof. Profit is maximised where the equilibrium output of firm 1 is equal to that of a

Stackelberg leader, which is $\frac{1}{2}(1-c)$. Thus by Lemma A.1. $\frac{\left(1-\gamma_{1}+\lambda_{1}\right)-\left(1+\gamma_{1}+\lambda_{1}\right) c}{3\left(1-\gamma_{1}\right)+\lambda_{1}}=\frac{1}{2}(1-c)$. Cross multiplying we obtain, $2\left(1-\gamma_{1}+\lambda_{1}\right)-2\left(1+\gamma_{1}+\lambda_{1}\right) c=(1-c)\left(3\left(1-\gamma_{1}\right)+\lambda_{1}\right)$, or $\lambda_{1}(2-2 c)-2 \gamma_{1}-2 \gamma_{1} c+2(1-c)=3\left(1-\gamma_{1}\right)(1-c)+\lambda_{1}(1-c)$. Hence $\lambda_{1}(1-c)=3\left(1-\gamma_{1}\right)$ $(1-c)-2(1-c)+2 \gamma_{1}(1+c)$, from which the result follows.

To understand this result it is useful to consider the special case where $\gamma_{1}=0$. Then equation (9) says $\lambda_{1}=1$, which implies that the manager will assign weight one to the possibility that the opponents will produce zero output and will himself produce the monopoly output. This is desirable, since in this example, the monopoly output coincides with the output of a Stackelberg leader, which is the most profitable output. Of course it is very unlikely that a manager would assign the decision-weight one to the possibility that the opponent will produce zero output. However it remains true that profit can be raised by delegating to a manager who is more optimistic than the owner. From the point of view of the owner, there is an additional advantage of hiring an optimistic manager. The more optimistic the manager the lower the 
value of $\alpha$ needed to induce the manager to work. This second effect also implies that it is advantageous to hire an optimistic manager. Altogether these reasons suggest why we might expect to see ambiguity-loving individuals in markets.

\subsection{Bertrand Oligopoly}

\subsubsection{Equilibrium without Ambiguity}

We shall now consider price (Bertrand) competition. Consider 2 firms producing heterogeneous goods, which are close (but not perfect) substitutes. Firm $i$ can produce at constant marginal and average cost, $k>0$. Firm $i$ charges price $p_{i}$ for its output. We assume that firm $i$ faces a linear demand curve: $D_{i}\left(p_{i}, p_{-i}\right)=\max \left\{0, a+b p_{-i}-c p_{i}\right\}, a, b, c>0, a>k, c>b$. The following result describes the equilibrium when firms choose prices simultaneously.

Proposition 4.4 In Nash equilibrium of the Bertrand model,

1. the reaction function of firm $i$ is given by

$$
p_{i}\left(p_{-i}\right)=\frac{a+b p_{-i}+c k}{2 c},
$$

2. both firms charge a price equal to, $\hat{p}=\frac{a+c k}{2 c-b}$.

Proof. The profits of firm $i$ are given by, $u_{i}=\left(p_{i}-k\right)\left(a+b p_{-i}-c p_{i}\right)$. The first order condition for profit maximisation is: $\frac{\partial u_{i}}{\partial p_{i}}=\left(a+b p_{-i}-c p_{i}\right)-c\left(p_{i}-k\right)=0$, from which part 1 ) follows. Let $\hat{p}$ denote the level of price charged by both firms in a symmetric Nash equilibrium then $\hat{p}=\frac{a+b \hat{p}+c k}{2 c}$, which implies part 2 .

Now suppose that firm 1 is a price leader and must set price first. Firm 2 then observes the price set by firm 1 and chooses its own price to maximise profit.

Proposition 4.5 If firm 1 is a price leader its optimal price is given by:

$$
p_{1}=\frac{2 a c+b(a+c k)}{2\left(2 c^{2}-b^{2}\right)}+\frac{k}{2} .
$$

The proof is contained in the appendix. With price leadership, firm 1 sets a price above the Bertrand equilibrium level since it takes into account the fact that when it raises price, this causes firm 2 to raise price as well. In this game there is a second mover advantage. Firm 2 makes higher profits since it can slightly undercut firm 1's price. 


\subsubsection{Equilibrium under Ambiguity}

Now assume that each firm perceives its rival's behaviour as ambiguous and has beliefs represented by neo-additive capacities. Suppose that each firm perceives the worst case to be where its rival reduces price to marginal cost. Hence we assume, a firm's strategy set is the interval $[k, K] \subseteq \mathbb{R}$ for some sufficiently high $K$. We shall assume the best case is perceived to be where the rival firm sets price equal to $K$. Given this interpretation, it seems reasonable to require that $K$ be above the Nash equilibrium price, i.e., $K>\frac{(a+c k)}{(2 c-b)}$. We require $a+b k-c K>0$ to ensure that demand is positive at all quantities in the firms' strategy sets. With these assumptions the (Choquet) expected profit of firm $i$ becomes: $V_{i}=\left(1-\gamma_{i}-\lambda_{i}\right)\left(p_{i}-k\right)\left(a+b p_{-i}-c p_{i}\right)+$ $\gamma_{i}\left(p_{i}-k\right)\left(a+b k-c p_{i}\right)+\lambda_{i}\left(p_{i}-k\right)\left(a+b K-c p_{i}\right)$. Simplifying $V_{i}=\left(p_{i}-k\right)\left(a-c p_{i}\right)$

$+\left(p_{i}-k\right) b\left[\left(1-\gamma_{i}-\lambda_{i}\right) p_{-i}+\gamma_{i} k+\lambda_{i} K\right]$. The first-order condition for profit maximisation is: $\frac{\partial V_{i}}{\partial p_{i}}=a-c p_{i}-c\left(p_{i}-k\right)+b\left[\left(1-\gamma_{i}-\lambda_{i}\right) p_{-i}+\gamma_{i} k+\lambda_{i} K\right]=0$. Thus firm $i$ 's reaction function is given by

$$
\rho^{i}\left(p_{-i}\right)=\frac{a+b\left[\left(1-\gamma_{i}-\lambda_{i}\right) p_{-i}+\gamma_{i} k+\lambda_{i} K\right]+c k}{2 c} .
$$

Equation (11) defines a non-singular system of linear equations, hence the Bertrand equilibrium is unique. Since $K \geqslant p_{-i} \geqslant k$, an increase in $\lambda_{i}$ (resp. $\gamma_{i}$ ) will shift firm $i$ 's reaction curve up (resp. down) and hence increase (resp. decrease) the equilibrium price. The price of firm $-i$ will also increase, since reaction curves slope upwards. Consider firm 1. An increase in optimism causes it to place more weight on good outcomes. In this context, a good outcome would be firm 2 charging a high price. Since the model exhibits strategic complementarity, this gives firm 1 an incentive to increase its price. This discussion is summarised in the following proposition.

Proposition 4.6 In Bertrand oligopoly an increase in optimism (resp. pessimism) of firm $i$ causes both firms to set higher (resp. lower) prices in equilibrium.

The case of two firms with symmetric linear demand functions is illustrated in figure 2. An increase in pessimism causes the best-response function to shift down and the slope to decrease. Firms have their own markets in which to react to the other's price. Uncertainty about the other price is equivalent to uncertainty about a firm's own demand. The lower a given firm sets the price, the smaller the market the opponents will face. Firms' concern about low demand in their respective market, provides an incentive for charging lower prices than in a conventional 
Figure 2: Bertrand equilibrium and uncertainty

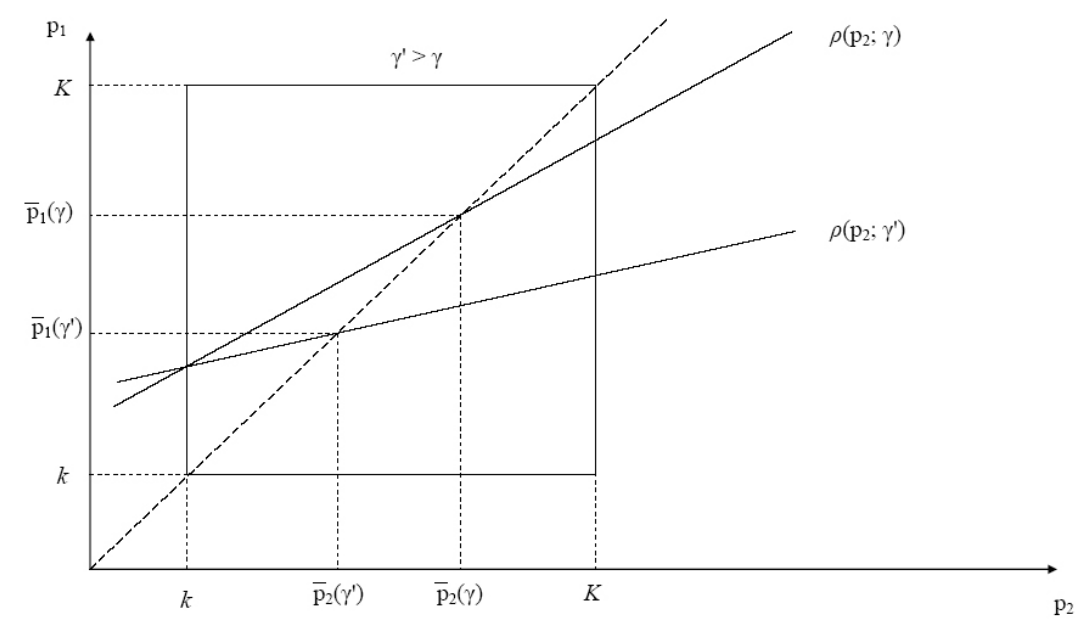

(Bertrand) equilibrium. Hence, pessimism tends to increase the competitiveness of Bertrand markets.

\subsubsection{Strategic Delegation}

Equation (11) shows that an increase in $\lambda_{i}$ will increase the equilibrium prices of both firms. In Bertrand oligopoly, a given firm can gain a strategic advantage by committing to price above the equilibrium level, see Fershtman and Judd (1987). This causes rivals to raise their prices, which gives the first firm an indirect benefit since its profits are higher the greater the prices of its rivals. Our results show that, appointing an optimistic manager would be a way to commit to a price above the Nash equilibrium level. Hence an optimistic manager will make more profit than an expected utility maximiser.

To illustrate the possibilities for strategic delegation we consider an specific form for the demand function.

Example 2 Assume $a=c=2$ and $b=1$, i.e., $D\left(p_{i}, p_{-i}\right)=2+p_{-i}-2 p_{i}$, for $i=1,2$. By Propositions 4.4 and 4.5 , the Nash equilibrium price is, $\hat{p}=\frac{2}{3}+\frac{2}{3} k$, while a price leader would charge $\tilde{p}_{1}=\frac{10+9 k}{14}$. We assume that firm 2 is a conventional profit maximising firm. Consider the case where firm 1 has ambiguity neutral owners, who delegate decision making to a manager, whose beliefs are represented by a neo-additive capacity. In this case we can show that $\gamma_{1}=0$, $\lambda_{1}=\frac{10-5 k}{56 K-38-37 k}$, are profit-maximising levels of $\lambda_{1}$ and $\gamma$. This claim is proved in Lemma A.2 
in the appendix.

The discussion of strategic delegation assumes that the ambiguity attitude of the manager is observable. Managers do make public speeches and reports, which could be used to indicate their ambiguity attitude. Many managers do appear to cultivate an optimistic view of their firm's performance. This could, in part, be motivated by strategic considerations.

In both Bertrand and Cournot oligopoly, profits can be increased above the Nash equilibrium level by employing an optimistic manager. This is unusual as most comparative static results are reversed when one moves from Cournot to Bertrand competition.

\section{$5 \quad$ Peace-making}

Interactive decision making under ambiguity is not confined to economic contexts. We believe that throughout social sciences there are many natural applications for our formal framework of ambiguity. To illustrate this point, we next consider a model of peace-making. This model also allows us to study the impact of ambiguity in a game with multiple equilibria ${ }^{12}$

\subsection{A Peace-making Game}

Consider two players $i=1,2$ interpreted as the parties involved in a conflict. Each player $i$ chooses a strategy $s_{i} \in S_{i}=[0,1]$. We interpret $s_{i}=0$ as no effort, whereas $s_{i}=1$ is full effort to peace-making. Higher values of $s_{i}$ correspond to greater peace-making efforts by individual $i$. We assume that benefits from peace-making have the following form:

$$
b\left(s_{1}, s_{2}\right)=\left\{\begin{array}{cll}
\varepsilon\left(s_{1}+s_{2}\right)^{2} & \text { if } \quad s_{1}<1 \text { or } s_{2}<1,0<\varepsilon<\frac{1}{4} \\
1 & \text { if } \quad s_{1}=s_{2}=1 .
\end{array}\right.
$$

The benefits from peace making are increasing in the efforts of both parties, hence there are positive externalities. An effort towards peace by one party brings benefits of peace to both. The benefit function is convex, which implies there is strategic complementarity in peace-making. The more effort is supplied by one party, the greater the marginal benefit of peace-making for the other. The discontinuity at $\langle 1,1\rangle$ indicates that there is a qualitative difference between peace and a war of very low intensity.

\footnotetext{
${ }^{12}$ This example also shows that continuous payoff functions are not necessary in Proposition 3.2
} 
Peace-making can be costly. For example, decision makers may face threats from extremists in their own camp not interested in peace. For simplicity we assume some linear costs $c s_{i}, c>0$. The payoff function $u$ of either party $i=1,2$ is written

$$
u_{i}\left(s_{i}, s_{j}\right)=b\left(s_{i}, s_{j}\right)-c s_{i} .
$$

The game is given by $G^{P}=\left\langle\left(S_{i}, u_{i}\right)_{i=1,2}\right\rangle$. Peace is viewed as a public good produced with increasing returns to scale. The following result characterises the Nash equilibria of the peace game. This is the benchmark without ambiguity.

Proposition 5.1 Solutions without ambiguity of the peace-making game $G^{P}$, are characterised as follows:

1. if $\varepsilon>c$, then full peace-making effort is the strictly dominant strategy for $i=1,2$;

2. if $1-\varepsilon \geqslant c \geqslant \varepsilon$ then there exist two Nash equilibria in pure strategies one with full peace-making effort and one where no effort is supplied by either party 13

3. if $c>1-\varepsilon$, then no effort is the strictly dominant strategy for $i=1,2$.

The proof is contained in the appendix.

Case (3) describes a situation where each side views the benefits of peace as being less than the costs of peace-making, regardless of what the other party does. Consequently peace is not established. Such a situation would arise if benefits from peace are small compared to costs of peace-making efforts. This does not seem a realistic representation of situations such as in the Middle East or Northern Ireland, where it seems most people perceive peace as worth achieving if possible.

Case (1) is the non-problematic case. Benefits from peace are strictly larger than the costs. Hence both parties provide full effort and peace is established. Again this does not appear to be a reasonable model of the world's conflict situations.

Case (2) is the interesting intermediate case. There are substantial benefits from peace but benefits from intermediate peace-making efforts are not enough to justify the costs. This seems to fit the circumstances in many potential conflict situations. Two Nash equilibria in pure

\footnotetext{
${ }^{13}$ There exists also a Nash equilibrium in mixed strategies where parties mix between zero and full peace-making effort.
} 
strategies arise, one in which both parties engage in full peace-making efforts establishing peace and one in which no effort is made and peace is not achieved.

\subsection{Peace-making under Ambiguity}

Now we study the impact of ambiguity on the peace-making game. The following proposition shows more optimism makes a successful peace process more likely. In the case where Nash equilibrium is not unique, ambiguity can play a role in equilibrium selection. If the degree of optimism is sufficiently high, there is a unique equilibrium in which the peace process succeeds. Pessimism has the opposite effect. If there is enough pessimism, then peace will not be established.

Proposition 5.2 The impact of ambiguity in the peace-making game, $G^{P}$, is as follows:

1. if $\varepsilon \geqslant c$, then any equilibrium under ambiguity involves only the Nash equilibrium strategy under certainty, i.e., $s_{i}=1, i=1,2$;

2. if $1-\varepsilon>c>\varepsilon$, then any equilibrium under ambiguity involves only strategies $s_{i}=1$ or $s_{i}=0, i=1,2$. Moreover, there exists $\bar{\lambda}$ (resp. $\bar{\gamma}$ ) such that if $\lambda \geqslant \bar{\lambda}($ resp. $\gamma \geqslant \bar{\gamma}$ ) then $s_{i}=1$ (resp. $s_{i}=0$ ) is the unique equilibrium strategy for $i=1,2$.

3. If $c \geqslant 1-\varepsilon$, then any equilibrium under ambiguity involves only the Nash equilibrium strategy under certainty, i.e., $s_{i}=0, i=1,2$.

The proof is contained in the appendix.

We believe that case (2) is the relevant one when peace-making poses a serious political problem. It is in this case, that ambiguity makes a difference. A high degree of pessimism causes peace-making efforts to break down. On the other hand optimism (or more confidence in the actions of the other side) can cause the peace-making process to be successful.

\section{General Results}

In this section we present some more general results for 2-player games with strategic complements. We consider a game with 2-players, $i=1,2$, where the strategy sets are subsets of the real line. In particular let $S_{i}=\left[\underline{s}_{i}, \bar{s}_{i}\right] \subseteq \mathbb{R}$ for $i=1,2$ and $S=S_{1} \times S_{2}$. Player $i$ has a 
concave utility function $u^{i}\left(s_{1}, s_{2}\right)$ and has beliefs on $S_{-i}$ represented by a neo-additive capacity $\nu_{i}=\lambda_{i}+\left(1-\lambda_{i}-\gamma_{i}\right) \pi_{i}$. The following assumption is maintained throughout this section.

Assumption 6.1 (Strict Concavity) For all $s_{1}, s_{2} \in S, u_{11}^{i}\left(s_{1}, s_{2}\right)<0, i=1,2$.

Proposition 6.1 (Uniqueness) Under Assumption 6.1 and positive or negative externalities, if the amount of ambiguity perceived by player $i, \lambda_{i}+\gamma_{i}$ is sufficiently large then the equilibrium under ambiguity is unique.

The proof is contained in the appendix. This result may be explained as follows. If player $i$ believes player - $i$ 's behaviour to be more ambiguous, player $i$ 's behaviour becomes less responsive to changes in $-i$ 's strategy. Thus the best-response function becomes flatter, which results in a unique equilibrium.

Next we investigate the comparative statics of changing ambiguity attitudes. To get unambiguous comparative static results we assume strategic complementarity.

Assumption 6.2 (Strategic Complementarity) The game G has strategic complements, if $u_{12}^{i}\left(s_{1}, s_{2}\right)>0$, for $i=1,2,14$

Strategic complementarity says that if player $-i$ increases his/her strategy this raises the marginal benefit to $i$ of increasing his/her own strategy. If player $i$ becomes more optimistic (s)he will place higher weight on good outcomes. If there are positive (resp. negative) externalities a good outcome will be interpreted as the opponent playing a high (resp. low) strategy. In the presence of strategic complementarity this gives $i$ an incentive to increase (resp. decrease) his/her strategy. If equilibrium is unique, an increase in optimism will increase (resp. decrease) equilibrium strategies of both players. If equilibrium is not unique we get a similar result. The set of equilibria increases (resp. decreases), in the sense that the strategies played in the highest and lowest equilibria increase (resp. decrease).

Proposition 6.2 (Comparative Statics) Under Assumptions 6.2 and positive (resp. negative) externalities the strategies of both players in the highest and lowest equilibria are increasing (resp. decreasing) functions of $\lambda_{1}$ and $\lambda_{2}$ and decreasing (resp. increasing) functions of $\gamma_{1}$ and $\left.\gamma_{2}\right)$.

\footnotetext{
${ }^{14}$ As usual $u_{12}^{i}$ denotes $\frac{\partial^{2} u^{i}}{\partial s_{1} \partial s_{2}}$.
} 
The proof is contained in the appendix. We obtain following corollary from Propositions 6.1 and 6.2 .

Corollary 6.1 (Equilibrium Selection) Under Assumptions 6.2 and positive (resp. negative) externalities, if $\lambda_{i}$ is sufficiently large, then equilibrium is unique and is larger (resp. smaller) than the largest (resp. smallest) equilibrium without ambiguity. If $\gamma_{i}$ is sufficiently large, then equilibrium is unique and is smaller (resp. larger) than the smallest (resp. largest) equilibrium without ambiguity.

Thus ambiguity can act as an equilibrium selection device. Under positive (resp. negative) externalities, if agents are sufficiently optimistic, all will focus on an equilibrium in which high (resp. low) strategies are played. The assumption of positive (resp. negative) externalities and strategic complementarity implies that the highest (resp. smallest) equilibrium is Pareto superior. In this case, optimism would select the equilibrium with the highest level of economic activity. As usual, pessimism has the opposite effect.

Without further assumptions, Proposition 6.2 can not directly be extended to games with strategic substitutes, i.e., games for which $u_{12}^{i}\left(s_{1}, s_{2}\right)<0$, even in the case of two players only. Although one can show that for negative (positive) externalities, best-response strategies increase (decrease) in the degree of optimism (and opposite for the degree of pessimism), the change of equilibrium depends on the relative shift of those best-responses correspondences.

\section{Concluding Discussion}

We introduce a simple model of ambiguity in strategic games and show how it can be applied to many situations of interest in economics and social sciences. New results are derived for both, optimistic as well as pessimistic attitudes towards ambiguity. It is possible to generalise these applications in many ways. The applications here were chosen to represent cases of strategic substitutes (Cournot equilibrium), strategic complements with a unique equilibrium (Bertrand equilibrium) and strategic complements with multiple equilibria (peace-making).

The assumptions imposed can be relaxed using lattice theoretical methods, see Topkis (1998). For instance, if the strategy space is a complete lattice, then existence results and results on the monotone comparative statics of equilibrium under ambiguity with respect to ambiguity attitudes can be derived if payoff functions are supermodular and have increasing differences. 
Extensions to more than two players are possible but introduce technical complications concerning the product capacity, see Eichberger and Kelsey (2000) and Eichberger and Kelsey (2002).

Lo (1996) and Marinacci (2000) present alternative equilibrium definitions based on different notions of support. In Lo's definition of equilibrium players do not perceive ambiguity about whether their opponents play best responses. This results in behavior which does not differ substantially from Nash equilibrium. There have been a number of definitions of support proposed for convex capacities. See Ryan (1997) for a full discussion. If $\lambda=0$ the neo-additive capacity is convex. Our definition of support coincides with most of the proposed definitions in this case.

Our model of ambiguity allows for a meaningful notion of optimism and pessimism. Wakker (2001) provides precise definitions of optimism and pessimism in CEU models. Epstein (1999) and Ghirardato and Marinacci (2002) provide alternative concepts of ambiguity aversion. In the case of CEU, Ghirardato and Marinacci take additive capacities as the benchmark case of no ambiguity-aversion, while Epstein argues for capacities which are monotone transformations of an additive probability (probabilistic sophistication) as the relevant benchmark. This implies that a convex and increasing transformation of an additive probability is interpreted as ambiguity-aversion by Ghirardato and Marinacci and as probabilistic risk-aversion by Epstein. We cannot resolve this controversy here. However, we mainly consider pure equilibria of games, in which case the two definitions agree.

Some related results can be found in Eichberger and Kelsey (2002). The present paper extends those results since they apply to optimistic as well as pessimistic attitudes to ambiguity. In addition the present paper has continuous rather than discrete strategy spaces. Eichberger and Kelsey (2002) confined attention to symmetric equilibria of symmetric games, assumptions not used in our paper. Moreover, Eichberger and Kelsey (2002) established uniqueness of equilibrium with a high degree of ambiguity when a restrictive assumption was satisfied (Assumption 3.2 of Eichberger and Kelsey (2002)). The results in Eichberger and Kelsey (2002) are proved for general pessimistic CEU preferences. This demonstrates that the restriction to neo-additive capacities is not crucial for our results but simplify greatly the technical exposition. Eichberger and Kelsey (2002) provide also some further applications. For example, in a model of voluntary contributions to public goods it is shown that ambiguity increases the provision of public goods. This potentially explains why donations often appear to be well above Nash equilibrium levels.

The applications, presented in this paper, can only serve to illustrate the type of results 
which one can expect to obtain by including ambiguity in economic analysis. Our intuition suggests that the conclusions obtained are not unreasonable. So far, there exists experimental evidence mainly for the impact of ambiguity aversion on individual decision making, Kilka and Weber (1998).

We believe that our approach is simple and intuitive in order to be applicable to various problems in economics and social sciences by a wide audience. For example, in a Cournot oligopoly model with ambiguity similar to the one of Section 4.1, Fontini (2005) finds that optimistic firms may benefit from the presence of pessimistic firms.

The theory in the present paper provides some testable hypotheses. For instance, for given externalities, ambiguity has the opposite effect in games of strategic complements and substitutes. In an experiment, Eichberger, Kelsey, and Schipper (2007), we found support for this hypothesis.

\section{A Appendix}

\section{A.1 Oligopoly}

Lemma A.1 Assume that the manager of firm 2 is ambiguity neutral (i.e., $\gamma_{2}=\lambda_{2}=0$ ), while the manager of firm 1 is not necessarily ambiguity neutral. Then under Cournot quantity competition the equilibrium output of firm 1 is given by:

$$
\bar{x}_{1}=\frac{\left(1-\gamma_{1}+\lambda_{1}\right)-\left(1+\gamma_{1}+\lambda_{1}\right) c}{3\left(1-\gamma_{1}\right)+\lambda_{1}} .
$$

Proof. From equation 77, firm 1's reaction function is given by, $R^{1}\left(x_{2}\right)=\frac{\left(1-\gamma_{1}\right)-\left(1-\gamma_{1}-\lambda_{1}\right) x_{2}-c}{2\left(1-\gamma_{1}\right)}$. By similar reasoning firm 2's reaction function is, $R^{2}\left(x_{1}\right)=\frac{1-c-x_{1}}{2}$. Solving for equilibrium in the usual way we obtain: $x_{1}=\frac{2\left(1-\gamma_{1}\right)-\left(1-\gamma_{1}-\lambda_{1}\right)\left(1-c-x_{1}\right)-2 c}{4\left(1-\gamma_{1}\right)}$. Thus $\left[\frac{4\left(1-\gamma_{1}\right)-\left(1-\gamma_{1}-\lambda_{1}\right)}{4\left(1-\gamma_{1}\right)}\right] x_{1}=\frac{\left(1-\gamma_{1}+\lambda_{1}\right)-\left(1+\gamma_{1}+\lambda_{1}\right) c}{4\left(1-\gamma_{1}\right)}$ from which the result follows.

Proof of Proposition 4.5 By equation 10, firm 2's reaction function is given by $p_{2}=\frac{a+b p_{1}+c k}{2 c}$. Firm 1's profits are given by, $u_{1}=\left(p_{1}-k\right)\left(a+b \frac{a+b p_{1}+c k}{2 c}-c p_{1}\right)$. The first order condition for profit maximisation is: $\frac{\partial u_{1}}{\partial p_{1}}=a+b \frac{a+c k}{2 c}+\left(\frac{b^{2}-2 c^{2}}{2 c}\right) p_{1}+\left(p_{1}-k\right)\left(\frac{b^{2}-2 c^{2}}{2 c}\right)=0$. Solving for $p_{1}$ we find, $2\left(\frac{2 c^{2}-b^{2}}{2 c}\right) p_{1}=$ $k\left(\frac{2 c^{2}-b^{2}}{2 c}\right)+a+b \frac{a+c k}{2 c}$, from which the result follows.

Lemma A.2 Provided $K \geqslant \frac{10+9 k}{14}$, in Example 2 the optimal value of $\lambda_{1}$ is

$$
\lambda_{1}=\frac{10-5 k}{56 K-38-37 k} \geqslant 0 .
$$


Proof. By equation 11, firm 2's reaction function is given by $p_{2}=\frac{2+p_{1}+2 k}{4}$. Firm 1's reaction function is given by $p_{1}=\frac{2+2 k+\lambda_{1} K+(1-\lambda) p_{2}}{4}$. Solving for equilibrium, $p_{1}=\frac{8+8 k+4 \lambda_{1} K+(1-\lambda)(2+2 k)}{16}+(1-\lambda) \frac{p_{1}}{16}$. Hence $\left[\frac{16-\left(1-\lambda_{1}\right)}{16}\right] p_{1}=\frac{8+8 k+4 \lambda_{1} K+\left(1-\lambda_{1}\right)(2+2 k)}{16}$ or $p_{1}=\frac{8+8 k+4 \lambda_{1} K+\left(1-\lambda_{1}\right)(2+2 k)}{16-\left(1-\lambda_{1}\right)}$.

Profit is maximised where this is equal to the price which would be chosen by a price leader without ambiguity. Hence $\frac{8+8 k+4 \lambda_{1} K+\left(1-\lambda_{1}\right)(2+2 k)}{16-\left(1-\lambda_{1}\right)}=\frac{10+9 k}{14}$. Solving for $\lambda_{1}, 112+112 k+56 \lambda_{1} K+$ $28\left(1-\lambda_{1}\right)(1+k)=160-10\left(1-\lambda_{1}\right)+144 k-9 k\left(1-\lambda_{1}\right)$, which implies $56 \lambda_{1} K-38 \lambda_{1}-37 \lambda_{1} k=10-5 k$, from which equation $(14)$ follows.

Note that $56 K-38-37 k \geqslant 56\left(\frac{10+9 k}{14}\right)-38-37 k=40+36 k-37 k=2-k \geqslant 0$, since $2=a>k$. (Recall $\frac{10+9 k}{14}$ is the output a price leader would choose.)

\section{A.2 Peace-making}

Proof of Proposition 5.1 Since $b$ is convex, any party's best response is either $s_{i}=0$ or $s_{i}=1$.

Case $[1]$ If $s_{2} \neq 1, u\left(1, s_{2}\right)-u\left(s_{1}, s_{2}\right)=\varepsilon\left(1+s_{2}\right)^{2}-c-\left[\varepsilon\left(s_{1}+s_{2}\right)^{2}-c s_{1}\right]$ $=\varepsilon\left(1-s_{1}^{2}+2 s_{2}-2 s_{1} s_{2}\right)-c\left(1-s_{1}\right)=\varepsilon\left(\left(1+s_{1}\right)\left(1-s_{1}\right)+2 s_{2}\left(1-s_{1}\right)\right)-c\left(1-s_{1}\right)$ $=\left(1-s_{1}\right)\left[\varepsilon\left(1+s_{1}+2 s_{2}\right)-c\right]>0$, since, by assumption, $\varepsilon>c$.

The case $s_{2}=1$ can be covered as follows: $u(1,1)-u\left(s_{1}, 1\right)=1-c-\left[\varepsilon\left(s_{1}+1\right)^{2}-c s_{1}\right]=$ $(1-4 \varepsilon)+4 \varepsilon-\varepsilon\left(s_{1}^{2}+2 s_{1}+1\right)-c\left(1-s_{1}\right)=(1-4 \varepsilon)+2 \varepsilon\left(1-s_{1}\right)+\varepsilon\left(1-s_{1}^{2}\right)-c\left(1-s_{1}\right)=(1-4 \varepsilon)+$ $\left(1-s_{1}\right)\left[2 \varepsilon+\varepsilon\left(s_{1}+1\right)-c\right]>0$ since $\varepsilon>c$.

Case (2) To show that $s_{1}=s_{2}=1$ is a Nash equilibrium, by convexity of $b$ it is enough to show $u(1,1) \geqslant u(0,1)$. This holds since, $u(1,1)-u(0,1)=1-\varepsilon-c \geqslant 0$. Now $u(0,0)=0, u(1,0)=\varepsilon-c \leqslant 0$, by assumption, which implies that $s_{1}=s_{2}=0$ is also a Nash equilibrium.

Case (3) If $s_{1} \neq 1, u\left(0, s_{2}\right)-u\left(s_{1}, s_{2}\right)=\varepsilon s_{2}^{2}-\varepsilon\left(s_{1}+s_{2}\right)^{2}+c s_{1}=c s_{1}-\varepsilon\left(2 s_{1} s_{2}+s_{1}^{2}\right) \geqslant c-3 \varepsilon>0$, since $c>1-\varepsilon$ implies $c>\frac{3}{4} \geqslant 3 \varepsilon$. The remaining case follows since $u(1,1)-u(0,1)=1-\varepsilon-c<0$.

Proof of Proposition 5.2 Cases (1) and (3) are proved by Proposition 5.1 and the observation that CEU preferences respect strict dominance.

Case (2) By convexity the only best responses can be 0 or 1 . Without loss of generality consider player 1 . Assume that his/her beliefs are represented by a neo-additive-capacity $\nu=\lambda+(1-\gamma-\lambda) \pi$. Let $V(1)$ (resp. $V(0))$ denote his/her (Choquet) expected utility if (s)he chooses 1 (resp. 0). Then, $V(1)=\lambda(1-c)+\gamma(\varepsilon-c)+(1-\gamma-\lambda)\left[\varepsilon \mathbf{E}_{\pi}\left(1+s_{2}\right)^{2}-c\right], V(0)=\lambda \varepsilon+(1-\gamma-\lambda) \varepsilon \mathbf{E}_{\pi} s_{2}^{2}$, where $\mathbf{E}_{\pi}$ denotes expectation with respect to the additive probability $\pi$. Now $V(1)-V(0)=\lambda(1-c)+\gamma(\varepsilon-c)+$ $(1-\gamma-\lambda)\left[\varepsilon \mathbf{E}_{\pi}\left(1+s_{2}\right)^{2}-c\right]-\lambda \varepsilon-(1-\gamma-\lambda) \varepsilon \mathbf{E}_{\pi} s_{2}^{2}=\lambda(1-\varepsilon-c)+\gamma(\varepsilon-c)+(1-\gamma-\lambda)\left[\varepsilon \mathbf{E}_{\pi}\left(1+2 s_{2}\right)-c\right]$. By assumption $1-\varepsilon-c>0$ and $\varepsilon-c<0$, hence if $\lambda$ (resp. $\gamma$ ) is sufficiently large $V(1)>V(0)$ (resp. $V(1)<V(0))$, from which the result follows. 


\section{A.3 General Results}

Lemma A.3 Under Assumption 6.1 and positive or negative externalities, the slope of the best-response functions is given by

$$
\begin{aligned}
& R^{1 \prime}\left(s_{2}\right)=\frac{-\left(1-\lambda_{1}-\gamma_{1}\right) u_{12}^{1}\left(R^{1}\left(s_{2}\right), s_{2}\right)}{\lambda_{1} M_{11}^{1}\left(R^{1}\left(s_{2}\right)\right)+\gamma_{1} m_{11}^{1}\left(R^{1}\left(s_{2}\right)\right)+\left(1-\lambda_{1}-\gamma_{1}\right) u_{11}^{1}\left(R^{1}\left(s_{2}\right), s_{2}\right)} \\
& R^{2 \prime}\left(s_{1}\right)=\frac{-\left(1-\lambda_{2}-\gamma_{2}\right) u_{12}^{2}\left(s_{1}, R^{2}\left(s_{1}\right)\right)}{\lambda_{2} M_{22}^{2}\left(R^{2}\left(s_{1}\right)\right)+\gamma_{2} m_{22}^{2}\left(R^{2}\left(s_{1}\right)\right)+\left(1-\lambda_{2}-\gamma_{2}\right) u_{22}^{2}\left(s_{1}, R^{2}\left(s_{1}\right)\right)} .
\end{aligned}
$$

Proof. Let $R^{i}$ denote the best-response function of player $i$.

By positive (resp. negative) externalities, we can set $M^{i}\left(s_{i}\right) \equiv u_{i}\left(s_{i}, \bar{s}_{-i}\right)\left(\operatorname{resp} . M^{i}\left(s_{i}\right) \equiv u_{i}\left(s_{i}, \underline{s}_{-i}\right)\right)$ and $m^{i}\left(s_{i}\right) \equiv u_{i}\left(s_{i}, \underline{s}_{-i}\right)$ (resp. $\left.m^{i}\left(s_{i}\right) \equiv u_{i}\left(s_{i}, \bar{s}_{-i}\right)\right)$ for any $s_{i} \in S_{i}$. Consider player 1 , his/her Choquet expected utility is given by: $\lambda_{1} M^{1}\left(s_{1}\right)+\gamma_{1} m^{1}\left(s_{1}\right)+\left(1-\lambda_{1}-\gamma_{1}\right) u^{1}\left(s_{1}, s_{2}\right)$. By Assumption 6.1 his/her best-response function is defined by,

$$
\lambda_{1} M_{1}^{1}\left(R^{1}\left(s_{2}\right)\right)+\gamma_{1} m_{1}^{1}\left(R^{1}\left(s_{2}\right)\right)+\left(1-\lambda_{1}-\gamma_{1}\right) u_{1}^{1}\left(R^{1}\left(s_{2}\right), s_{2}\right)=0 .
$$

Differentiating 15. with respect to $s_{2}$ we obtain, $\lambda_{1} M_{11}^{1}\left(R^{1}\left(s_{2}\right)\right) R^{1 \prime}\left(s_{2}\right)+\gamma_{1} m_{11}^{1}\left(R^{1}\left(s_{2}\right)\right) R^{1 \prime}\left(s_{2}\right)+$ $\left(1-\lambda_{1}-\gamma_{1}\right) u_{11}^{1}\left(R^{1}\left(s_{2}\right), s_{2}\right) R^{1 \prime}\left(s_{2}\right)+\left(1-\lambda_{1}-\gamma_{1}\right) u_{12}^{1}\left(R^{1}\left(s_{2}\right), s_{2}\right)=0$. From which the result follows. The slope of $R^{2}$ can be derived by analogous reasoning.

Proof of Proposition 6.1 Consider the function, $g: S^{1} \times S^{2} \rightarrow S^{1} \times S^{2}$, defined by $g\left(s_{1}, s_{2}\right)=$ $\left\langle R^{1}\left(s_{2}\right)-s_{1}, R^{2}\left(s_{1}\right)-s_{2}\right\rangle$. The partial derivatives of $g$ are $\frac{\partial g^{1}}{\partial s_{1}}=-1, \frac{\partial g^{1}}{\partial s_{2}}=R^{1 \prime}\left(s_{2}\right), \frac{\partial g^{2}}{\partial s_{1}}=R^{2 \prime}\left(s_{1}\right)$ and $\frac{\partial g^{2}}{\partial s_{2}}=-1$. Let $J$ denote the Jacobian matrix of $g$. Then $J=\left(\begin{array}{cc}-1 & R^{1 \prime}\left(s_{2}\right) \\ R^{2 \prime}\left(s_{1}\right) & -1\end{array}\right)$. The trace of $J$ is -2 . Thus if the determinant of $J$ is positive, both eigenvalues must be negative and hence $J$ is negative definite. The determinant of $J$ is $1-R^{1 \prime}\left(s_{2}\right) R^{2 \prime}\left(s_{1}\right) \geqslant 1-\frac{\left(1-\lambda_{2}-\gamma_{2}\right)\left(1-\lambda_{1}-\gamma_{1}\right) Q^{2}}{\delta^{2}}$, since $R^{1 \prime}\left(s_{2}\right)=\frac{-\left(1-\lambda_{1}-\gamma_{1}\right) u_{12}^{1}\left(R^{1}\left(s_{2}\right), s_{2}\right)}{\lambda_{1} M_{11}^{1}\left(R^{1}\left(s_{2}\right)\right)+\gamma_{1} m_{11}^{1}\left(R^{1}\left(s_{2}\right)\right)+\left(1-\lambda_{1}-\gamma_{1}\right) u_{11}^{1}\left(R^{1}\left(s_{2}\right), s_{2}\right)},\left|R^{1 \prime}\left(s_{2}\right)\right| \leqslant \frac{\left(1-\lambda_{1}-\gamma_{1}\right) Q}{\delta}$, where $Q=\max _{\left\langle s_{1}, s_{2}\right\rangle \in S}\left|u_{12}^{1}\left(s_{1}, s_{2}\right)\right|$ and $\delta=\min _{\left\langle s_{1}, s_{2}\right\rangle \in S}\left|u_{11}^{1}\left(s_{1}, s_{2}\right)\right|$. Since the strategy space is compact this minimum exists. It follows that $J$ is negative definite if $\lambda_{1}+\gamma_{1}$ is sufficiently large. By Theorem 4.3 of Eichberger (1993), negative definiteness of $J$ implies that equilibrium is unique.

The next result characterises extremal equilibria in terms of the slope of the best-response functions.

Lemma A.4 If the highest and lowest equilibria are interior equilibria, then $R^{1 \prime}\left(s_{2}\right) R^{2 \prime}\left(s_{1}\right) \leqslant 1$ at these equilibria.

Proof. Define $\rho:\left[\underline{s}_{1}, \bar{s}_{1}\right] \rightarrow\left[\underline{s}_{1}, \bar{s}_{1}\right]$ by $\rho\left(s_{1}\right)=R^{1}\left(R^{2}\left(s_{1}\right)\right)$. By assumption there are no corner equilibria, hence $\rho\left(\underline{s}_{1}\right)>\underline{s}_{1}$ and $\rho\left(\bar{s}_{1}\right)<\bar{s}_{1}$. Let $\left\langle\hat{s}_{1}, \hat{s}_{2}\right\rangle$ be an equilibrium such that $R^{1 \prime}\left(s_{2}\right) R^{2 \prime}\left(s_{1}\right)>1$. Then for all sufficiently small $\delta>0, \rho\left(\hat{s}_{1}+\delta\right)>\hat{s}_{1}+\delta$. Let $\phi\left(s_{1}\right)=\rho\left(s_{1}\right)-s_{1}$. Then $\phi\left(\hat{s}_{1}+\delta\right)>0$ and $\phi\left(\bar{s}_{1}\right)<0$. By the intermediate value theorem, there exists $s^{\prime} \in\left(\hat{s}_{1}+\delta, \bar{s}_{1}\right)$ such that $\phi\left(s_{1}^{\prime}\right)=s_{1}^{\prime}$. Therefore $\left\langle\hat{s}_{1}, \hat{s}_{2}\right\rangle$ is not the highest equilibrium. Similar arguments apply to the lowest equilibrium. 
Proof of Proposition 6.2

Let $\left\langle\hat{s}_{1}, \hat{s}_{2}\right\rangle$ denote the highest equilibrium. Assume first that $\left\langle\hat{s}_{1}, \hat{s}_{2}\right\rangle$ is an interior equilibrium. Since $\left\langle\hat{s}_{1}, \hat{s}_{2}\right\rangle$ is an interior equilibrium it satisfies the first order conditions for best responses.

$$
\begin{aligned}
& \lambda_{1} M_{1}^{1}\left(s_{1}\right)+\gamma_{1} m_{1}^{1}\left(s_{1}\right)+\left(1-\lambda_{1}-\gamma_{1}\right) u_{1}^{1}\left(s_{1}, s_{2}\right)=0 \\
& \lambda_{2} M_{2}^{2}\left(s_{2}\right)+\gamma_{2} m_{2}^{2}\left(s_{2}\right)+\left(1-\lambda_{2}-\gamma_{2}\right) u_{2}^{2}\left(s_{1}, s_{2}\right)=0 .
\end{aligned}
$$

Differentiating (17) with respect to $\lambda_{1}$ we obtain:

$$
\begin{gathered}
{\left[\lambda_{2} M_{22}^{2}\left(s_{2}\right)+\gamma_{2} m_{22}^{2}\left(s_{2}\right)+\left(1-\lambda_{2}-\gamma_{2}\right) u_{22}^{2}\left(s_{1}, s_{2}\right)\right] \frac{\partial s_{2}}{\partial \lambda_{1}}+\left(1-\lambda_{2}-\gamma_{2}\right) u_{12}^{2}\left(s_{1}, s_{2}\right) \frac{\partial s_{1}}{\partial \lambda_{1}}=0 . \text { Hence }} \\
\frac{\partial s_{2}}{\partial \lambda_{1}}=R^{2 \prime}\left(s_{1}\right) \frac{\partial s_{1}}{\partial \lambda_{1}} .
\end{gathered}
$$

Differentiating (16) with respect to $\lambda_{1}$ we obtain,

$\left[\lambda_{1} M_{11}^{1}\left(s_{1}\right)+\gamma_{1} m_{11}^{1}\left(s_{1}\right)+\left(1-\lambda_{1}-\gamma_{1}\right) u_{11}^{1}\left(s_{1}, s_{2}\right)\right] \frac{\partial s_{1}}{\partial \lambda_{1}}+\left(1-\lambda_{1}-\gamma_{1}\right) u_{12}^{1}\left(s_{1}, s_{2}\right) \frac{\partial s_{2}}{\partial \lambda_{1}}=u_{1}^{1}\left(s_{1}, s_{2}\right)-$ $M_{1}^{1}\left(s_{1}\right)$. Hence $\frac{\partial s_{1}}{\partial \lambda_{1}}+\frac{\left(1-\lambda_{1}-\gamma_{1}\right) u_{12}^{1}\left(s_{1}, s_{2}\right)}{\lambda_{1} M_{11}^{1}\left(s_{1}\right)+\gamma_{1} m_{11}^{1}\left(s_{1}\right)+\left(1-\lambda_{1}-\gamma_{1}\right) u_{11}^{1}\left(s_{1}, s_{2}\right)} \frac{\partial s_{2}}{\partial \lambda_{1}}=\frac{u_{1}^{1}\left(s_{1}, s_{2}\right)-M_{1}^{1}\left(s_{1}\right)}{\lambda_{1} M_{11}^{1}\left(s_{1}\right)+\gamma_{1} m_{11}^{1}\left(s_{1}\right)+\left(1-\lambda_{1}-\gamma_{1}\right) u_{11}^{1}\left(s_{1}, s_{2}\right)}$. Substituting from 18, $\frac{\partial s_{1}}{\partial \lambda_{1}}-R^{1 \prime}\left(s_{2}\right) R^{2 \prime}\left(s_{1}\right) \frac{\partial s_{1}}{\partial \lambda_{1}}=\frac{u_{1}^{1}\left(s_{1}, s_{2}\right)-M_{1}^{1}\left(s_{1}\right)}{\lambda_{1} M_{11}^{1}\left(s_{1}\right)+\gamma_{1} m_{11}^{1}\left(s_{1}\right)+\left(1-\lambda_{1}-\gamma_{1}\right) u_{11}^{1}\left(s_{1}, s_{2}\right)}$. Hence $\frac{\partial s_{1}}{\partial \lambda_{1}}=$ $\frac{u_{1}^{1}\left(s_{1}, s_{2}\right)-M_{1}^{1}\left(s_{1}\right)}{\lambda_{1} M_{11}^{1}\left(s_{1}\right)+\gamma_{1} m_{11}^{1}\left(s_{1}\right)+\left(1-\lambda_{1}-\gamma_{1}\right) u_{11}^{1}\left(s_{1}, s_{2}\right)}\left[1-R^{1 \prime}\left(s_{2}\right) R^{2 \prime}\left(s_{1}\right)\right]^{-1}$. If the game has positive (resp. negative) externalities, then $u_{1}^{1}\left(s_{1}, s_{2}\right)-M_{1}^{1}\left(s_{1}\right)=u_{1}^{1}\left(s_{1}, s_{2}\right)-u_{1}^{1}\left(s_{1}, \bar{s}_{2}\right)\left(\right.$ resp. $\left.=u_{1}^{1}\left(s_{1}, s_{2}\right)-u_{1}^{1}\left(s_{1}, \underline{s}_{2}\right)\right)$. By Assumption 6.2. if the game has positive (resp. negative) externalities then $u_{1}^{1}\left(s_{1}, s_{2}\right)-M_{1}^{1}\left(s_{1}\right)<1$ (resp. > 1). By Lemma A.4 $1-R^{1 \prime}\left(s_{2}\right) R^{2 \prime}\left(s_{1}\right) \geqslant 0$. Hence, if the game has positive (resp. negative) externalities then $\frac{\partial s_{1}}{\partial \lambda_{1}} \geqslant 0$ (resp. $\leqslant 0$ ), and by equation $18 \frac{\partial s_{2}}{\partial \lambda_{1}} \geqslant 0$ (resp. $\left.\leqslant 0\right)$. The results for the effect of changing the other parameters on extremal equilibria can be obtained by analogous reasoning.

Now consider the case where the highest equilibrium is on the boundary of the strategy set and the game has positive externalities. In particular suppose that when $\lambda_{1}=\tilde{\lambda}_{1}$ that the highest equilibrium is $\left\langle\bar{s}_{1}, \bar{s}_{2}\right\rangle$. Firstly it is trivially true that a decrease in $\lambda_{1}$ must (weakly) decrease the equilibrium strategies of both players. Now suppose $\lambda_{1}$ increases from $\tilde{\lambda}_{1}$ to $\hat{\lambda}_{1}$. The equilibrium at $\left\langle\bar{s}_{1}, \bar{s}_{2}\right\rangle$ satisfies the Kuhn-Tucker conditions:

$$
\begin{aligned}
& \tilde{\lambda}_{1} M_{1}^{1}\left(\bar{s}_{1}\right)+\gamma_{1} m_{1}^{1}\left(\bar{s}_{1}\right)+\left(1-\tilde{\lambda}_{1}-\gamma_{1}\right) u_{1}^{1}\left(\bar{s}_{1}, \bar{s}_{2}\right) \geqslant 0 \\
& \lambda_{2} M_{1}^{2}\left(\bar{s}_{2}\right)+\gamma_{2} m_{1}^{2}\left(\bar{s}_{2}\right)+\left(1-\lambda_{2}-\gamma_{2}\right) u_{1}^{2}\left(\bar{s}_{1}, \bar{s}_{2}\right) \geqslant 0 .
\end{aligned}
$$

Since $\hat{\lambda}_{1} M_{1}^{1}\left(\bar{s}_{1}\right)+\gamma_{1} m_{1}^{1}\left(\bar{s}_{1}\right)+\left(1-\hat{\lambda}_{1}-\gamma_{1}\right) u_{1}^{1}\left(\bar{s}_{1}, \bar{s}_{2}\right)=\tilde{\lambda}_{1} M_{1}^{1}\left(\bar{s}_{1}\right)+\gamma_{1} m_{1}^{1}\left(\bar{s}_{1}\right)+\left(1-\tilde{\lambda}_{1}-\gamma_{1}\right) u_{1}^{1}\left(\bar{s}_{1}, \bar{s}_{2}\right)$, the Kuhn-Tucker conditions are still satisfied when $\lambda_{1}=\hat{\lambda}_{1}$. By concavity, these conditions are sufficient hence $\left\langle\bar{s}_{1}, \bar{s}_{2}\right\rangle$ remains the highest equilibrium when $\lambda_{1}=\hat{\lambda}_{1}$. Analogous reasoning applies to the lowest equilibrium, other parameter changes and negative externalities. 


\section{References}

Bewley, T. F. (2002): "Knightian Decision Theory: Part 1," Decisions in Economics and Finance, 25, 79-110.

Camerer, C., And M. Weber (1992): "Recent Developments in Modelling Preferences: Uncertainty and Ambiguity," Journal of Risk and Uncertainty, 5, 325-370.

Chateauneuf, A., J. Eichberger, And S. Grant (2007): "Choice under Uncertainty with the Best and Worst on Mind: NEO-Additive Capacities," Journal of Economic Theory, forthcoming.

Choquet, G. (1953-4): "Theory of Capacities," Annales Institut Fourier, 5, 131-295.

Cohen, M. (1992): "Security Level, Potential Level, Expected Utility: A Three Criteria Decision Model Under Risk," Theory and Decision, 33, 101-104.

Cohen, M., J.-Y. Jaffray, And T. SAID (1985): "Individual Behaviour under Risk and Uncertainty: An Experimental Study," Theory and Decision, 18, 203-228.

Cooper, A., W. Dunkelberg, and C. Woo (1988): "Entrepreneur's perceived Chances of Success," Journal of Business Venturing, 3, 97-108.

Dow, J., And S. R. C. Werlang (1994): "Nash Equilibrium under Uncertainty: Breaking Down Backward Induction," Journal of Economic Theory, 64, 305-324.

Eichberger, J. (1993): Game Theory for Economists. Academic Press, San Diego.

Eichberger, J., And D. Kelsey (2000): "Non-Additive Beliefs and Strategic Equilibria," Games and Economic Behavior, 30, 183-215.

(2002): "Strategic Complements, Substitutes and Ambiguity: The Implications for Public Goods," Journal of Economic Theory, 106, 436-466.

Eichberger, J., D. Kelsey, And B. C. Schipper (2007): "Granny versus Game Theorist: Ambiguity in Experimental Games," Theory and Decision, forthcoming.

Ellsberg, D. (1961): "Risk, Ambiguity and the Savage Axioms," Quarterly Journal of Economics, 75, 643-669.

Epstein, L. G. (1999): "A Definition of Uncertainty Aversion," Review of Economic Studies, 66, 579606.

Fershtman, C., And K. L. Judd (1987): "Equilibrium Incentives in Oligopoly," American Economic Review, 77, 927-940. 
Fontini, F. (2005): "Cournot Oligopoly under Strategic Uncertainty with Optimistic and Pessimistic Firms," Metroeconomica, 56, 318-333.

Ghirardato, P., And M. Marinacci (2002): "Ambiguity Made Precise: A Comparative Foundation," Journal of Economic Theory, 102, 251-289.

GilboA, I. (1987): "Expected Utility with Purely Subjective Non-additive Probabilities," Journal of Mathematical Economics, 16, 65-88.

GilboA, I., And D. Schmeidler (1989): "Maxmin Expected Utility with a Non-Unique Prior," Journal of Mathematical Economics, 18, 141-153.

Glicksberg, I. (1952): "A further Generalization of the Kakutani fixed point theorem with application to Nash equilibrium points," Proceedings of the National Academy of Sciences, 38, 170-174.

Gonzalez, R., And G. Wu (1999): "On the Shape of the Probability Weighting Function," Cognitive Psychology, 38, 129-166.

Hurwicz, L. (1951): "Optimality Criteria for Decision Making under Ignorance," Discussion Paper 370, Cowles Commission.

Kelsey, D. (1994): "Maxmin Expected Utility and Weight of Evidence," Oxford Economic Papers, 46, 425-444.

KILKA, M., AND M. Weber (1998): "What Determines the Shape of the Probability Weighting Function under Uncertainty?," Management Science, 47, 1712-1726.

Klieman, A. (1999): Constructive Ambiguity in Middle East Peace-Making. The Tami Steinmetz Center for Peace Research, Tel Aviv University, Tel Aviv.

Knight, F. H. (1921): Risk, Uncertainty, and Profit. Houghton Mifflin, New York.

Lo, K. C. (1996): "Equilibrium in Beliefs under Uncertainty," Journal of Economic Theory, 71, 443-484.

Lopes, L. (1987): "Between Hope and Fear: The Psychology of Risk," in Advances in Experimental Social Psychology, ed. by L. Berkowitz, pp. 255-295. Academic Press, New York.

MarinaCCI, M. (2000): "Ambiguous Games," Games and Economic Behavior, 31, 191-219.

Osborne, M. J., And A. Rubinstein (1994): A Course in Game Theory. MIT, Cambridge, MA.

RYAn, M. J. (1997): "CEU Preferences and Game Theoretic Equilibrium," The University of Auckland, working paper series no. $16 \%$.

Sarin, R., And P. Wakker (1992): "A Simple Axiomatization of Non-Additive Expected Utility," Econometrica, 60, 1255-1272. 
SARIn, R., AND P. WAKker (1998): "Revealed Likelihood and Knightian Uncertainty," Journal of Risk and Uncertainty, 16, 223-250.

Savage, L. J. (1954): Foundations of Statistics. Wiley, New York.

Scherer, F. (1970): Industrial Market Structure and Economic Performance. Rand McNally, Chicago.

Schmeidler, D. (1989): "Subjective Probability and Expected Utility without Additivity," Econometrica, $57,571-587$.

Seligman, M., And P. Schulman (1986): "Explanatory Style as a Predictor of Productivity and Quitting among Life Insurance Sales Agents," Journal of Personality and Social Psychology, 22, 93121.

Topkis, D. M. (1998): Supermodularity and Complementarity. Princeton University Press, New Jersey.

Vickers, J. (1985): "Delegation and the Theory of the Firm," Economic Journal, 95 (conference supplement), $138-147$.

Wakker, P. (2001): "Testing and Characterizing Properties of Nonadditive Measures Through Violations of the Sure Thing Principle," Econometrica, 69, 1039-1060. 